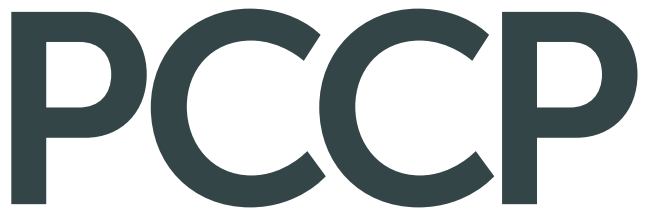

Physical Chemistry Chemical Physics www.rsc.org/pccp

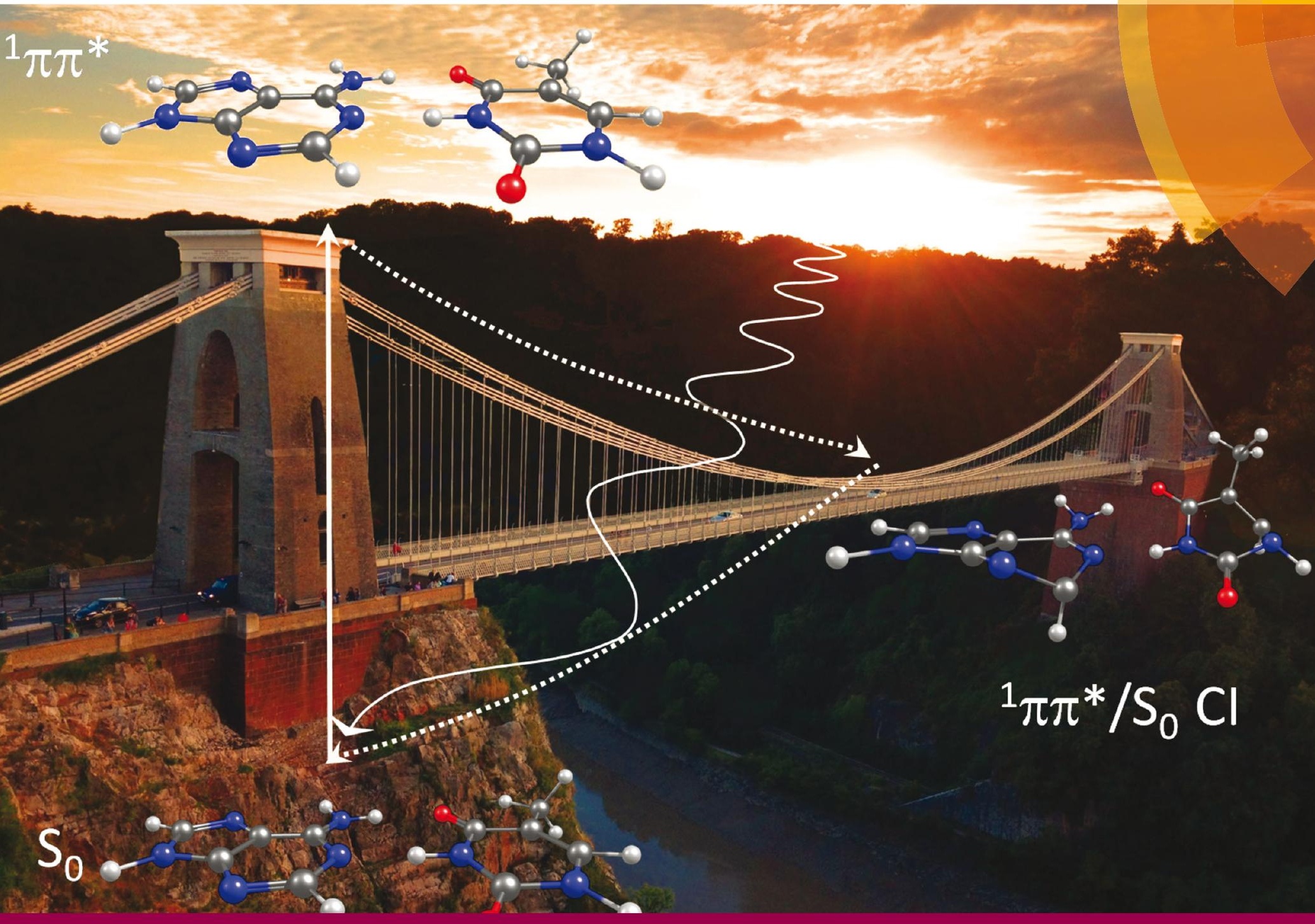

Themed issue: Prebiotic Chemistry

ISSN 1463-9076

\section{PERSPECTIVE}

Tolga N. V. Karsili, Michael N. R. Ashfold et al.

A 'bottom up', ab initio computational approach to understanding

fundamental photophysical processes in nitrogen containing heterocycles,

DNA bases and base pairs

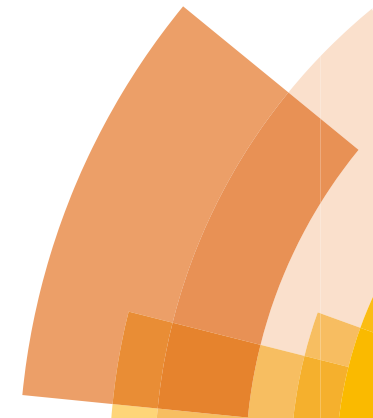




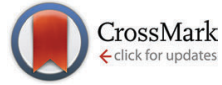

Cite this: Phys. Chem. Chem. Phys., 2016, 18, 20007

Received 9th January 2016 Accepted 7th March 2016

DOI: $10.1039 / c 6 c p 00165 c$

www.rsc.org/pccp

\title{
A 'bottom up', ab initio computational approach to understanding fundamental photophysical processes in nitrogen containing heterocycles, DNA bases and base pairs $\uparrow$
}

\author{
Barbara Marchetti, ${ }^{a}$ Tolga N. V. Karsili, ${ }^{a b}$ Michael N. R. Ashfold ${ }^{* a}$ and \\ Wolfgang Domcke ${ }^{b}$
}

\begin{abstract}
The availability of non-radiative decay mechanisms by which photoexcited molecules can revert to their ground electronic state, without experiencing potentially deleterious chemical transformation, is fundamental to molecular photostability. This Perspective Article combines results of new $a b$ initio electronic structure calculations and prior experimental data in an effort to systematise trends in the non-radiative decay following UV excitation of selected families of heterocyclic molecules. We start with the prototypical uni- and bicyclic molecules phenol and indole, and explore the structural and photophysical consequences of incorporating progressively more nitrogen atoms within the respective ring structures en route to the DNA bases thymine, cytosine, adenine and guanine. For each of the latter, we identify low energy non-radiative decay pathways via conical intersections with the ground state potential energy surface accessed by out-of-plane ring deformations. This is followed by summary descriptions and illustrations of selected rival (electron driven $\mathrm{H}$ atom transfer) non-radiative excited state decay processes that demand consideration once the nucleobases are merely components in larger biomolecular systems like nucleosides, and both individual and stacked base-pairs.
\end{abstract}

\section{Introduction}

Many recent experimental and theoretical studies have sought to explore the photophysics of simple heteroatom containing aromatic molecules $^{1-7}$ like phenols ${ }^{8-28}$ and azoles ${ }^{29-39}$ following excitation in the ultraviolet (UV) spectral region. Such systems are often termed prototypical, on the basis that a thorough understanding of their response to photoexcitation might well offer a useful guide to the photochemistry and photophysics of more complex and more biologically relevant molecules. Detailed understanding of their deactivation processes following UV excitation has thus long been seen as paramount. The first

\footnotetext{
${ }^{a}$ School of Chemistry, University of Bristol, Cantock's Close, Bristol, BS8 1TS, UK. E-mail: tolga.karsili@bristol.ac.uk, mike.ashfold@bristol.ac.uk; Tel: +44 (o)117928 8312

${ }^{b}$ Department of Chemistry, Technische Universität München, Lichtenbergstr. 4, 85748 Garching, Germany

$\dagger$ Electronic supplementary information (ESI) available: More details regarding the computational methods used; PECs along the relevant $Q_{\text {oop }}$ coordinate for all molecules displayed in Fig. 1 and 2; depictions of the active space orbitals used for the CASSCF and CASPT2 calculations; dominant orbital excitations that contribute to the first three singlet excited states of all molecules featured in Fig. 1-3 and 4(a); and PECs for the ground and first few singlet excited states of adenosine along $Q_{\text {oop }}$ calculated at the $\operatorname{ADC}(2) / c c-p V D Z$ level of theory. See DOI: $10.1039 / \mathrm{c} 6 \mathrm{cp} 00165 \mathrm{c}$
}

few excited states of these molecules can be partitioned in several different ways. One distinction is obvious; any given orbital promotion will support both singlet and triplet excited states. Unless stated otherwise, this Perspective focuses solely on the decay of singlet excited state molecules, since intersystem crossing to triplet states is not viewed as a significant decay process for the molecules and the (generally isolated molecule) conditions featured in this Perspective.

The nature of the orbital populated by photoexcitation is another key distinguisher. The highest occupied molecular orbital (HOMO) in the ground electronic $\left(\mathrm{S}_{0}\right)$ state of these molecules ranges between a (bonding) ring-centred $\pi$ orbital or a (largely non-bonding) $\mathrm{n}$ orbital centred on the heteroatom, whereas the orbital populated by photoexcitation may be a (ring-centred, anti-bonding) $\pi^{*}$ orbital or a $\sigma^{*}$ orbital localised on the heteroatom - to which we return later. $\pi^{*} \leftarrow \pi$ transitions typically have the largest absorption cross-sections and dominate the UV absorption spectrum; the diabatic ${ }^{1} \pi \pi^{*}$ states that result are often termed optically 'bright'. $\pi^{*} \leftarrow \mathrm{n}$ transitions are generally weaker, but much of the recent interest ${ }^{1-5,7}$ in these molecules has centred on the (hitherto often neglected) excited states formed by $\sigma^{*} \leftarrow \pi$ (or $\sigma^{*} \leftarrow \mathrm{n}$ ) promotions which, for brevity, we will henceforth describe simply as ${ }^{1} \pi \sigma^{*}$ excited states. The ${ }^{1} \pi \sigma^{*}$ states generally have much smaller absorption 
cross-sections and, as a result, are sometimes described as optically 'dark'.

The energetic ordering of the first excited ${ }^{1} \pi \sigma^{*},{ }^{1} \mathrm{n} \pi^{*}$ and ${ }^{1} \pi \pi^{*}$ states (i.e. the $1^{1} \pi \sigma^{*}, 1^{1} n \pi^{*}$ and $1^{1} \pi \pi^{*}$ states) is molecule dependent. In the case of azoles like pyrrole ${ }^{1,40}$ and imidazole, ${ }^{41-43}$ the $1^{1} \pi \sigma^{*}$ state is lowest in energy, whereas in the phenols ${ }^{2}$ the $1^{1} \pi \pi^{*}$ state is the lowest energy excited state in the FranckCondon (FC) region. The $\sigma^{*}$ orbital in these first row heterocycles has significant Rydberg (3s) character in the FC region, but acquires progressively more anti-bonding character upon $\mathrm{X}-\mathrm{H}(\mathrm{X}=\mathrm{N}, \mathrm{O}$, etc. $)$ bond extension. As a result, given the relative $1^{1} \pi \sigma^{*} \leftarrow S_{0}$ excitation and $\mathrm{X}-\mathrm{H}$ bond dissociation energies, the potential energy surfaces (PESs) for these $1^{1} \pi \sigma^{*}$ states are repulsive with respect to increasing $R_{\mathrm{X}-\mathrm{H}}$. Exciting molecules like imidazole at long UV wavelengths populates the $1^{1} \pi \sigma^{*}$ excited state directly and results in prompt $\mathrm{N}-\mathrm{H}$ bond fission. Long wavelength excitation of phenol, in contrast, populates the $1^{1} \pi \pi^{*}$ state which, at the diabatic level, is bound but can predissociate by coupling with the $1^{1} \pi \sigma^{*}$ state and subsequently breaking the $\mathrm{O}-\mathrm{H}$ bond on a much longer timescale. ${ }^{16,19}$

Bond fission is just one of the several possible decay pathways available to the excited state molecules considered in this Perspective. Almost all of these pathways involve radiationless transfer from the initial state populated by photoexcitation (e.g. between different ${ }^{1} \pi \pi^{*}$ and/or ${ }^{1} n \pi^{*}$ states, from a ${ }^{1} n \pi^{*}$ or ${ }^{1} \pi \pi^{*}$ state to a ${ }^{1} \pi \sigma^{*}$ state, or from a ${ }^{1} n \pi^{*}$ or ${ }^{1} \pi \pi^{*}$ state to the $S_{0}$ state). As discussed below, the strength of such couplings maximises at molecular geometries where the relevant PESs become near degenerate - so called regions of conical intersection (CI).

The DNA/RNA bases illustrate this progressive increase in complexity nicely. These molecules show strong $\pi^{*} \leftarrow \pi$ absorptions at wavelengths $\sim 260 \mathrm{~nm},{ }^{44,45}$ excitation of which could potentially induce photoreactions and harmful structural changes. Yet the DNA bases and base pairs display welldocumented photostability. ${ }^{46-53}$ This resistance to photodamage is attributable to ultrafast and efficient non-radiative (and nonreactive) relaxation to the $S_{0}$ state via internal conversion (IC). Femtosecond time-resolved studies of the isolated bases - in the gas phase $\mathrm{e}^{54-65}$ and in aqueous solution ${ }^{66-77}$ - return excitation energy and environment dependent excited state lifetimes but, in all cases, these lifetimes are short; radiationless transfer to the $\mathrm{S}_{0}$ state in the isolated bases typically occurs on a (sub)picosecond time scale. $A b$ initio calculations attribute such efficient excited state decay to the presence of low energy CIs between the excited $\left({ }^{1} \pi \pi^{*}\right)$ and $S_{0}$ state PESs along out-of-plane deformation (ring puckering) coordinates. ${ }^{78-107}$

Here we seek to explain, and to systematise, various of the non-radiative excited state decay processes available to related families of nitrogen containing heterocycles by pooling new results obtained using a range of $a b$ initio electronic structure methods with existing literature data. Without question, the existing literature contains reports of more comprehensive and detailed computational studies of several of the systems covered in this Perspective, and we have sought to cite these at appropriate points in the narrative. The prime focus of the
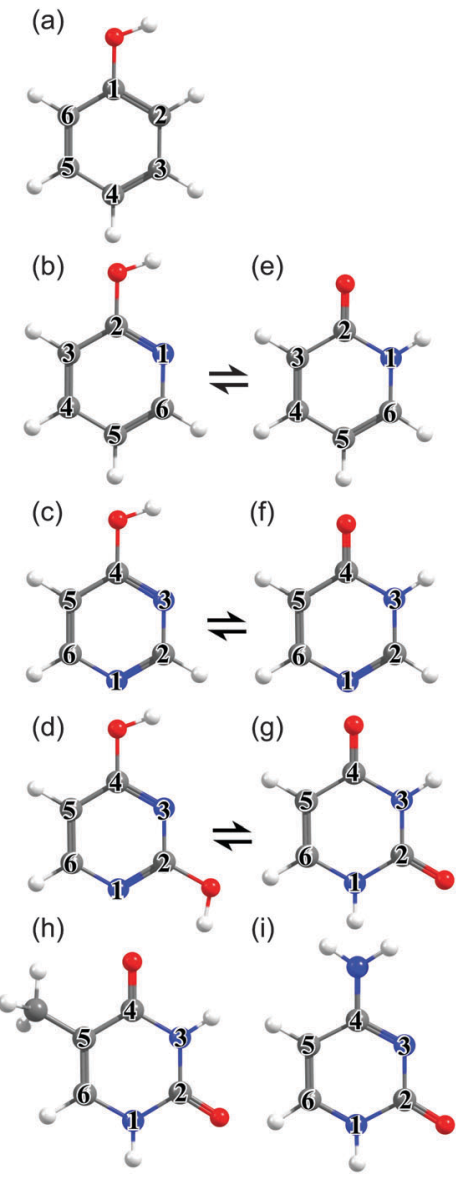

Fig. 1 Unicyclic molecules considered in this work: (a) phenol, (b) 2-hydroxypyridine, (c) 4-hydroxypyrimidine, (d) 2,4-dihydroxypyrimidine, (e) 2(1H)-pyridone, (f) $4(3 H)$-pyrimidone, (g) uracil $(\mathrm{U})$, (h) thymine (T) and (i) cytosine $(\mathrm{C})$, with the ring atoms numbered for future reference.

present study is the systematic analysis of the excited state photophysics of families of related molecules investigated in an (as best as possible) even handed and self-consistent manner.

The philosophy of the study is as follows. Uracil (U), thymine (T) and cytosine (C) are pyrimidine derivatives, and hydroxypyridines and hydroxypyrimidines relate to phenol if one or more of the ring $\mathrm{C}$ atoms are replaced by $\mathrm{N}$ atoms. Adenine (A) and guanine (G) are purine derivatives. Purine itself can be viewed as the result of substituting three of the ring $\mathrm{C}$ atoms in indole by $\mathrm{N}$ atoms, and indole comprises imidazole fused with a benzene ring. This Perspective thus starts by considering trends in the excited state photophysics of the family of unicyclic molecules shown in Fig. 1, wherein $\mathrm{C}$ atoms in phenol are progressively replaced by $\mathrm{N}$ atoms, leading through selected pyridines and pyrimidines en route (after appropriate tautomerism and/or derivitization) to $\mathrm{U}, \mathrm{T}$ and $\mathrm{C}$. This is followed by a similar analysis of the evolving excited state photophysics of the family of bicyclic molecules shown in Fig. 2 as we step from indole through two azaindoles and purine to A and G. Both studies serve to illustrate the progressive lowering of the energies of ${ }^{1} \pi \pi^{*} / \mathrm{S}_{0}$ CIs in out-of-plane deformation coordinates brought about by incorporating $\mathrm{N}$ atoms in the ring. The Perspective concludes by 
<smiles>CC1[B]C(C)C(C)C(C)C1C</smiles>

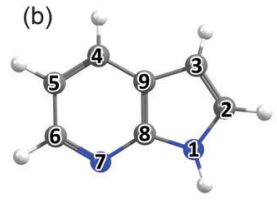

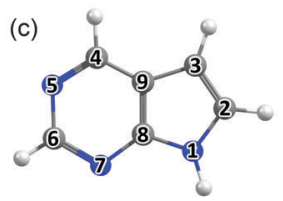

(e)<smiles>COC1CCCCC1B1CB(C)C(C)C2CCCCC12</smiles>

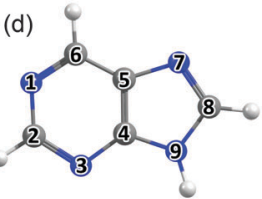

(f)

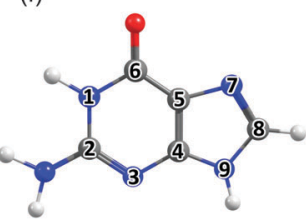

Fig. 2 Bicyclic molecules considered in this work: (a) indole, (b) 7-azaindole, (c) 5,7-azaindole, (d) $9 \mathrm{H}$-purine, (e) adenine (A) and (f) guanine (G), with the ring atoms numbered for future reference. (a)

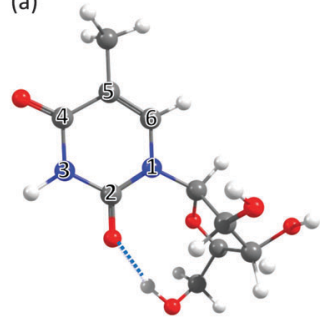

(c)

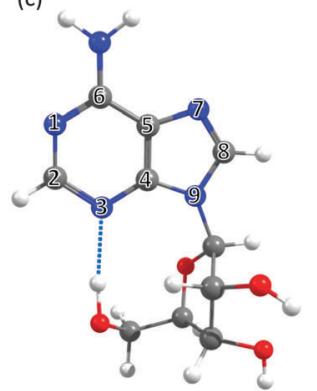

(b)

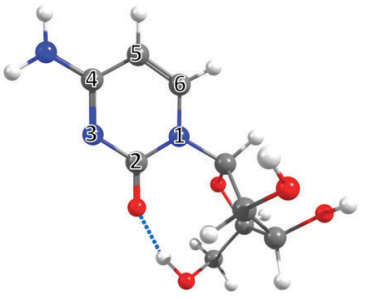

(d)

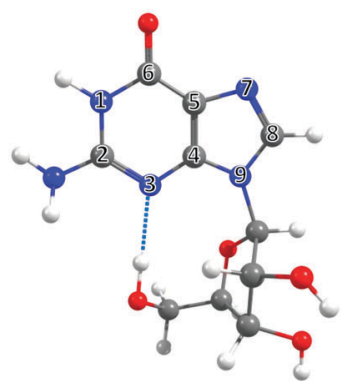

Fig. 3 The nucleosides: (a) 5-methyluridine, (b) cytidine, (c) adenosine and (d) guanosine, with the ring atoms of the base numbered for future reference.

highlighting additional non-radiative decay pathways (most notably excited state $\mathrm{H}$ atom transfer processes) that demand consideration as the molecular size and complexity increases further - as exemplified by the ribonucleosides (Fig. 3) and the base-pairs (Fig. 4).

\section{Theoretical methodology}

A brief overview of the ab initio computational methods used in the present calculations is given here. Fuller details can be found in the ESI. $\dagger$
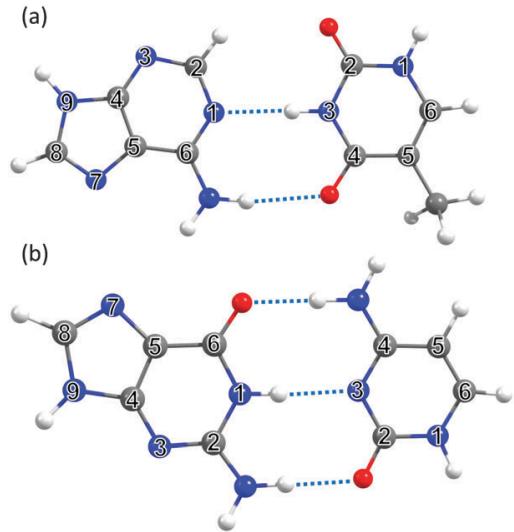

Fig. 4 The DNA and RNA base pairs: (a) adenine-thymine (A-T) and (b) guanine-cytosine $(G-C)$, with the ring atoms in the respective bases again numbered for future reference.

\subsection{Ground state tautomerism}

Using the Gaussian09 computational package, ${ }^{108}$ the ground state tautomers were assessed by optimising, where appropriate, the enol and keto forms along with the transition state (TS) connecting the two structures using Møller-Plesset second order perturbation theory (MP2) along with Dunning's augmented correlation consistent basis set of triple- $\xi$ quality (AVTZ). ${ }^{109}$ The minimum energy path (MEP) for tautomerism was computed at the DFT/CAM-B3LYP/AVTZ level of theory using the intrinsic reaction coordinate (IRC) algorithm embedded within Gaussian 09.

\subsection{Individual bases}

The geometries of the ground state minimum, all conical intersections between the $S_{0}$ and ${ }^{1} \pi \pi^{*}$ PESs in the out-of-plane distortion coordinate, $Q_{\text {oop}}$, and (where appropriate) any TSs of interest were optimised using Complete Active Space-Self Consistent Field (CASSCF) methods coupled with the 6-31G(d) basis set using Gaussian09. ${ }^{110}$ All possible out-of-plane deformation $\mathrm{S}_{0} /{ }^{1} \pi \pi^{*}$ CIs were initially optimised. In each case, attention then focussed on the minimum energy CI (MECI) and subsequent linear interpolations in internal coordinates (LIICs) were constructed between the $S_{0}$ minimum and this MECI. The energies along this LIIC were then calculated using Complete Active Space with second-order Perturbation Theory (CASPT2) with a cc-pVDZ (VDZ) or AVDZ basis set, using the MOLPRO v2010.1 computational package. ${ }^{111}$ For the specific cases of phenol, 2-hydroxypyridine and 2-pyridone, unrelaxed CASPT2/ AVDZ scans were also calculated along the $R_{\mathrm{X}-\mathrm{H}}(\mathrm{X}=\mathrm{O}$ or $\mathrm{N})$ coordinate.

\subsection{Nucleosides and base pairs}

The $\mathrm{S}_{0}$ state geometries of the nucleosides were optimised using MP2/6-31G(d) whilst the $\mathrm{S}_{0} /{ }^{1} \pi \pi^{*}$ CI geometries were optimised using CASSCF/6-31G(d). MP2/6-31G(d) and CASSCF/ 6-31G(d) were also used to optimise the geometries of the $S_{0}$ minimum and the $S_{0} /{ }^{1} \pi \pi^{*} \mathrm{CI}$, respectively, for the A-T base pair. All MP2 and CASSCF calculations were carried out using Gaussian09. In each case, potential energy profiles along the 
LIIC linking these optimised critical points were constructed at the CASPT2/VDZ level of theory using the MOLPRO v2010.1 computation package. Relaxed potential energy profiles along the electron-driven proton (hydrogen) transfer (EDPT) coordinate of current interest were calculated for these four nucleosides and the A-T and G-C base pairs using ADC(2)/VDZ in Turbomole v6. $3^{112}$ for both the $S_{0}$ and $S_{1}\left({ }^{1} n \pi^{*}\right.$ (charge transfer (CT))) states. In the nucleosides, the driving coordinate of interest in each case was the terminal ribose sugar $\mathrm{O}-\mathrm{H}$ bond whereas, in the base pairs, it was the individual base centred $\mathrm{O}-\mathrm{H}$ or $\mathrm{N}-\mathrm{H}$ bonds.

\subsection{Radical-anion pairs}

Calculations on the $\mathrm{G}-\mathrm{C}$ and $\mathrm{A}-\mathrm{T}$ radical-anion base pairs were performed in order to mimic inter-stack electron transfer. The relaxed ground state potential energy profiles of the (adeninethymine) ${ }^{-}$and (guanine-cytosine) ${ }^{-}$radical-anion base pairs were computed at the TD-DFT/CAM-B3LYP/VDZ level of theory using $R_{\mathrm{N}-\mathrm{H}}$ as the driving coordinate in Gaussian09.

\section{Results and discussion}

The material in this section is structured as follows: several of the target molecules can exist as both keto- and enol-tautomers, and Section 3.1 reviews how substituting $\mathrm{C}$ atoms in the ring by $\mathrm{N}$ atoms affects the relative stabilities of these tautomers in the $\mathrm{S}_{0}$ state. The rest of the Perspective focusses on excited states. Sections 3.2 and 3.3 explore how the stabilities and nonradiative decay dynamics of the families of molecules displayed in Fig. 1 and 2 are influenced by $\mathrm{C} \leftrightarrow \mathrm{N}$ atom substitution within the ring. Sections 3.4 and 3.5 then explore some of the additional non-radiative decay pathways ( $\mathrm{H}$ atom transfer processes) that can arise in the selected nucleosides and base pairs shown in Fig. 3 and 4.

\subsection{Ground state tautomerism}

Table 1 compares the ground state energies of 2-hydroxypyridine, 4-hydroxypyrimidine and 2,4-dihydroxypyrimidine with those of the corresponding keto-tautomers calculated at several different levels of theory. The respective stabilities listed in Table 1 are all referenced to the more stable tautomer.

2-Hydroxypyridine/2-pyridone. As Table 1 shows, all levels of theory investigated suggest that 2-hydroxypyridine (i.e. the enol form) is the more stable tautomer $[\operatorname{CCSD}(\mathrm{T})$ $\left.\Delta E_{\text {enol-keto }} \sim 390 \mathrm{~cm}^{-1}\right]$ - in accord with gas phase experimental measurements. ${ }^{113}$ As Fig. 5(a) shows, the DFT/CAM-B3LYP/AVTZ calculations return a large $\left(\sim 12000 \mathrm{~cm}^{-1}\right)$ barrier to tautomerism in the isolated molecule. The greater relative stability calculated for the enol tautomer is consistent with its larger number of resonance structures over which the $\pi$ electron density can be delocalised. In solution, however, the relative stabilities of the two tautomers are very sensitive to the solvent polarity; in aqueous solution, for example, the stabilisation from hydrogen bonding ensures that the keto-form is the majority tautomer. ${ }^{114,115}$

4-Hydroxypyrimidine/4-pyrimidone. Adding another $\mathrm{N}$ atom within the six-membered ring stabilises the keto tautomer to the extent that, in the isolated molecule, 4-pyrimidone is calculated (and observed ${ }^{116,117}$ ) to be slightly the more stable tautomer - as shown in Table 1 and Fig. 5(b). This can be understood by recognising that, in the context of these heterocycles, the $\mathrm{N}$ atom is an electron donor. The $\mathrm{C}=\mathrm{O}$ group in the keto form is able to accept and stabilise additional $\pi$ density introduced in the 1-position, whereas an $\mathrm{OH}$ group in the 4-position (as in the enol form) would tend to be destabilised.

2,4-Dihydroxypyrimidine/uracil. Two independent ground state hydrogen transfer coordinates were explored for these tautomers, illustrated by the red and blue curves in Fig. 5(c) and (d). The calculations clearly show that uracil (structure 3d) is the most stable tautomer (consistent with experimental observation $^{118}$ ), followed by the hemi-keto tautomers (structures $3 \mathrm{~b}$ and 3c), with the fully enol tautomer (structure 3a) being the least stable - for the same electronic reasons as outlined above.

Cytosine, adenine and guanine. Ground state tautomerism is also possible in the other pyrimidine species shown in Fig. 1 (i.e. thymine (Fig. 1(h)) and cytosine (Fig. 1(i))) and in the various purines depicted in Fig. 2. For brevity, we restrict discussion to the DNA bases $\mathrm{C}, \mathrm{G}$ and $\mathrm{A}$. The distinguishing feature of these molecules ( $c f$. those discussed above) is the amino group, which affords additional possibilities of amino-imino tautomerism

Table 1 Relative stabilities $\left(\Delta E\right.$, in $\left.\mathrm{cm}^{-1}\right)$ of the enol $(E)$-keto $(K)$ tautomers of 2-hydroxypyridine/2-pyridone, 4-hydroxypyrimidine/4-pyrimidone and 2,4-dihydroxypyrimidine/uracil in their respective $S_{0}$ states, calculated at many levels of theory and quoted relative to that of the more stable tautomer (indicated in parentheses), along with relevant experimental data (where available)

\begin{tabular}{|c|c|c|c|c|c|}
\hline \multirow[b]{3}{*}{ Level of theory } & \multirow{3}{*}{$\begin{array}{l}\text { 2-Hydroxypyridine/2-pyridone } \\
\Delta E / \mathrm{cm}^{-1}(1 \mathrm{a}-1 \mathrm{~b})\end{array}$} & \multirow{3}{*}{$\begin{array}{l}\text { 4-Hydroxypyrimidine/4-pyrimidone } \\
\Delta E / \mathrm{cm}^{-1}(2 \mathrm{~b}-2 \mathrm{a})\end{array}$} & \multicolumn{3}{|c|}{ 2,4-Dihydroxypyrimidine/uracil } \\
\hline & & & \multicolumn{3}{|c|}{$\Delta E / \mathrm{cm}^{-1}$} \\
\hline & & & $(3 d-3 a)$ & $(3 d-3 c)$ & $(3 d-3 b)$ \\
\hline DFT/CAM-B3LYP/AVDZ & $110(\mathrm{E})$ & $240(\mathrm{~K})$ & $4090(\mathrm{~K})$ & $4000(\mathrm{~K})$ & $3830(\mathrm{~K})$ \\
\hline DFT/CASSCF(10,8)/AVDZ & $100(\mathrm{E})$ & $690(\mathrm{~K})$ & - & - & - \\
\hline MP2/AVTZ & $840(\mathrm{E})$ & $260(\mathrm{E})$ & 3370 (K) & $3760(\mathrm{~K})$ & $3470(\mathrm{~K})$ \\
\hline DF-MP2-F12/AVTZ & $800(\mathrm{E})$ & $230(\mathrm{E})$ & $3450(\mathrm{~K})$ & $3780(\mathrm{~K})$ & $3530(\mathrm{~K})$ \\
\hline CCSD/AVTZ & $420(\mathrm{E})$ & $120(\mathrm{~K})$ & $3890(\mathrm{~K})$ & $4050(\mathrm{~K})$ & $3650(\mathrm{~K})$ \\
\hline $\operatorname{CCSD}\left(\mathrm{F} 12^{*}\right) / \mathrm{AVTZ}$ & $410(\mathrm{E})$ & $130(\mathrm{~K})$ & $3970(\mathrm{~K})$ & $4080(\mathrm{~K})$ & $3710(\mathrm{~K})$ \\
\hline CCSD(T)(F12*)/AVTZ & $390(\mathrm{E})$ & $100(\mathrm{~K})$ & $3710(\mathrm{~K})$ & 3860 (K) & $3550(\mathrm{~K})$ \\
\hline CASPT2(12,11)/AVTZ & $1130(\mathrm{E})$ & $390(\mathrm{~K})$ & - & - & - \\
\hline Experiment & $270(\mathrm{E})^{112}$ & $167(\mathrm{~K})^{115,116}$ & - & - & $(K)^{117}$ \\
\hline
\end{tabular}



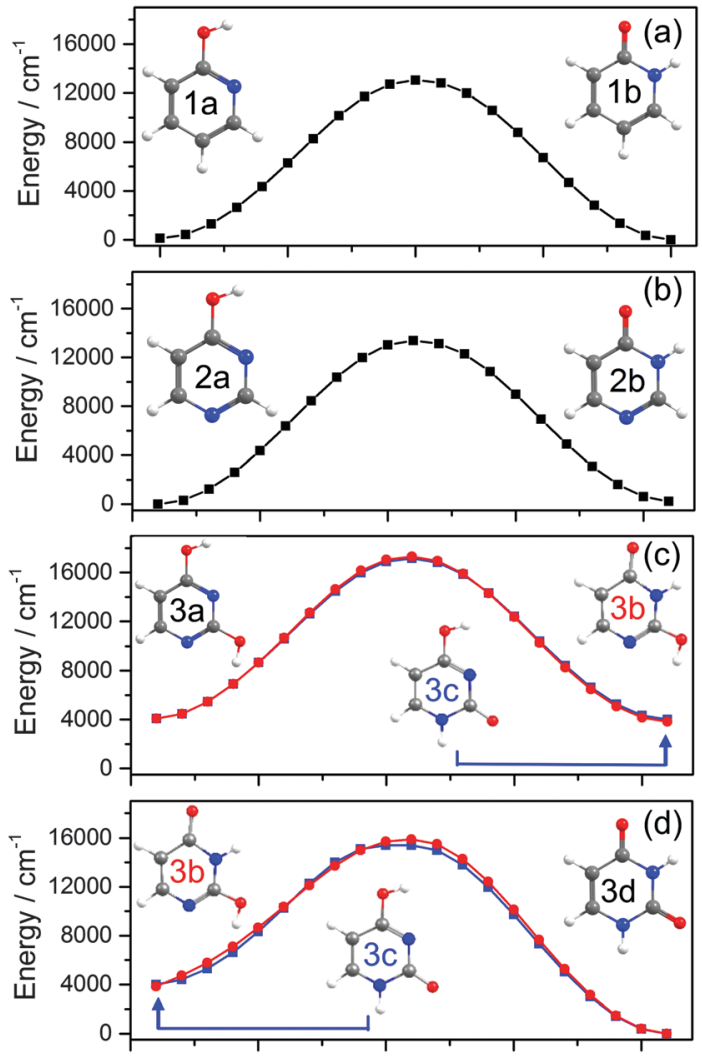

Enol / Keto Tautomerism coordinate

Fig. 5 Ground state MEP along the enol/keto tautomerism IRC calculated for (a) 2-hydroxypyridine/2-pyridone, (b) 4-hydroxypyrimidine/4(3H)-pyrimidone and (c) and (d) 2,4-dihydroxypyrimidine/uracil. The red curves in (c) and (d) show the calculated MEP for the sequence 2,4-dihydroxypyrimidine (i.e. the 2,4-dienol tautomer) to the $2(3 \mathrm{H})$-enol-keto form (structure $3 \mathrm{~b}$ ) and on to the $1 \mathrm{H}, 3 \mathrm{H}$-diketo form, uracil (structure $3 \mathrm{~d}$ ), while the blue curves map the potential for the same 2,4-dienol to uracil rearrangement but via the intermediate $4(1 H)$-enol-keto tautomer (structure $3 c$ ). The energies of structures $3 a-c$ are defined relative to the most stable structure (3d). and leads to a significant increase in the number of possible tautomers.

In cytosine, for example, there are fourteen possible tautomer/ rotamers, five of which are shown in Fig. 6(a)-(e). The $1 H$-ketoamino form (Fig. 6(a)) is the preferred tautomer in the WatsonCrick (WC) base pairing. $\mathrm{H}$ atom migration from the $\mathrm{N} 1-\mathrm{H}$ group to the neighbouring $\mathrm{O}$ atom or to the $\mathrm{N} 3$ atom gives rise to three distinct tautomers - two 2-enol-amino structures (of which only the anti-rotamer is shown in Fig. 6(b)) and the $3 H$-keto-amino tautomer (Fig. 6(c)). The relative stabilities of the different tautomeric forms are again sensitive to the chemical environment. Microwave, resonance enhanced multiphoton ionisation (REMPI) and core level photoemission measurements reveal both the $1 H$-keto-amino and 2-enol-amino tautomers in jet-cooled gas phase samples. ${ }^{119-122}$ Low temperature matrix isolation studies (in the IR and the UV) also identify both of these tautomers and conclude that the 2-enol-amino tautomer is more abundant, ${ }^{123,124}$ whereas the $1 H$-keto-amino form is most stable in aqueous solution. ${ }^{125}$ The available theoretical predictions are broadly supportive of these conclusions. ${ }^{126-128}$ Under isolated molecule conditions, the $1 H$-keto-amino and 2-enol-amino tautomers are calculated to lie lowest in energy (with the former $\sim 500 \mathrm{~cm}^{-1}$ more stable than the latter), with the two imino (Fig. 6(d) and (e)) and the $3 H$-keto-amino (Fig. 6(c)) tautomers consistently higher in energy. Micro-solvation with water molecules, under isolated gas-phase conditions, ${ }^{127}$ is predicted to favour the keto-amino tautomers, particular the $1 H$-keto-amino structure (calculated as being $\sim 2040 \mathrm{~cm}^{-1}$ more stable than the $3 \mathrm{H}$-equivalent) and to destabilise the enol and imino tautomers.

In $\mathrm{A}$, the $\mathrm{H}$ atom at the $\mathrm{N} 9$ position can migrate to any of the other nitrogen atoms within the ring, giving a total of fourteen possible tautomers/rotamers. ${ }^{129}$ Experimental studies have identified the presence of the $9 H$-amino (Fig. 6(f)) and, in some cases, the $7 \mathrm{H}$-amino (Fig. $6(\mathrm{~g}))$ tautomers in the gas phase, ${ }^{130-133}$
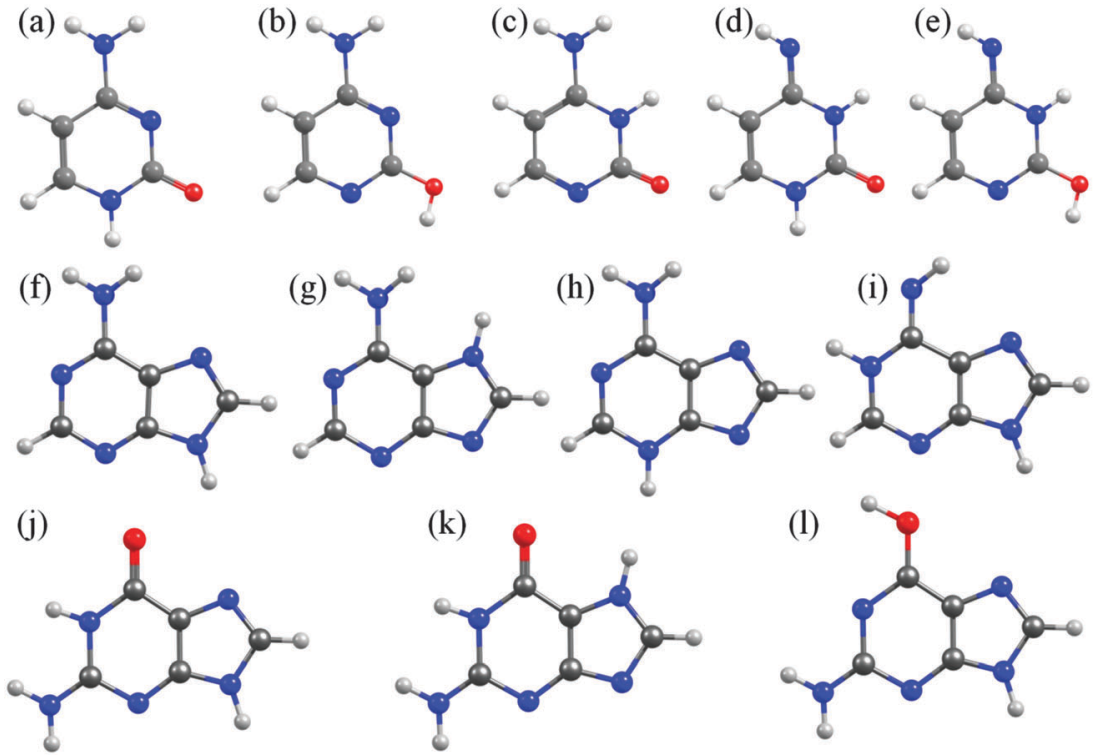

Fig. 6 Selected low energy tautomeric forms for cytosine (structures (a)-(e)), adenine (structures (f)-(i)) and guanine (structures (j)-(l)). 
in matrix isolation conditions ${ }^{134}$ and in polar solvent environments (e.g. water). ${ }^{135-138}$ All of these studies concur that the canonical $9 \mathrm{H}$-amino form is the dominant tautomer in all environments (as it is in DNA itself), though the $7 \mathrm{H}$-amino variant gains some relative stabilisation in polar solvents by virtue of its larger dipole moment. These experimental conclusions have been reinforced by numerous ab initio studies, though most predict a significant $\left(\sim 2400-3000 \mathrm{~cm}^{-1}\right)$ energy difference between the $9 H^{-}$and $7 H$-amino tautomers..$^{130,139,140}$ The $3 H$-aminoand the various imino forms (e.g. the $1 \mathrm{H}, 9 \mathrm{H}$-imino structure shown in Fig. 6(i)) are all calculated to be considerably higher in energy.

$\mathrm{G}$ is functionalized with both amino and keto groups, and has the greatest number of possible tautomers. ${ }^{141}$ An early IR matrix isolation study showed $G$ present as a mixture of enol and keto tautomers, ${ }^{142}$ and more recent studies have identified three different tautomers (one enol and two keto forms) in a supersonic jet. ${ }^{143-146}$ Guided by complementary ab initio calculations, these were assigned to the $1 \mathrm{H}, 7 \mathrm{H}$-keto-amino (Fig. 6(k)), the $1 \mathrm{H}, 9 \mathrm{H}$-keto-amino (Fig. 6(j)) and the $9 \mathrm{H}$-enolamino (Fig. 6(l)) tautomers, with the latter likely present as two different rotamers. Theory ${ }^{140,145-148}$ also predicts that the $1 \mathrm{H}, 7 \mathrm{H}$-keto-amino structure is the most stable as an isolated molecule, followed by the $1 H, 9 H$-keto-amino tautomer (with reported energy separations in the range $20-250 \mathrm{~cm}^{-1}$ ) and then the $9 H$-enol-amino tautomer (some $270-620 \mathrm{~cm}^{-1}$ above the global minimum structure). All of the other tautomers not shown in Fig. 6 are predicted to lie at least $1000 \mathrm{~cm}^{-1}$ above the global minimum. Once again, however, this energetic ordering is disturbed by solvating with water, ${ }^{146}$ under which circumstances the tautomeric equilibria are predicted to shift in favour of the $3 H, 7 H$-keto-amino (now the most stable), $7 H, 9 H$-keto-amino and $3 \mathrm{H}, 9 \mathrm{H}$-keto-amino tautomers.

The primary aim of this Perspective is to seek trends and differences in the excited state photophysics exhibited by these molecules. Thus we choose to focus attention on the tautomeric forms adopted by the various nucleobases in the common WC or in the Hoogsteen base pairing schemes (i.e. 1H-cytosine, $9 H$-adenine and $1 \mathrm{H}, 9 \mathrm{H}$-guanine, as depicted in Fig. 1(i), $2(\mathrm{e})$ and (f)).

Nucleosides. The tautomeric equilibria in the nucleosides are assumed not to differ substantially from those found with the individual bases, apart from the obvious cases where $\mathrm{N} 1$ (in the pyrimidines) or N9 (in the purines) is terminated by the pentose sugar and some of the tautomeric variety associated with $\mathrm{H}$ migration between the various $\mathrm{N}$ sites in the isolated monomers is obviously precluded.

\subsection{Unicyclic ring systems and the pyrimidine bases}

Vertical excitation energies. Table 2 lists vertical excitation energies (VEEs) calculated for the two lowest singlet excited states of the molecules depicted in Fig. 1 (with the character of the dominant orbital promotion indicated in parentheses). Fig. 7 shows the orbital promotions involved in forming the excited states of 2-hydroxypyridine, 2-pyridone and 7-azaindole; similar figures for the other systems considered in this Perspective are shown in the ESI. $\dagger$ The adiabatic excitation energies (AEEs) calculated for the respective first excited states generally agree well with the available experimental values.

As discussed in Section 3.1, the molecules formed by introducing one (or more) $\mathrm{N}$ atoms into the ring can exist as enol and keto tautomers. The present calculations show the $S_{1}$ and $\mathrm{S}_{2}$ states of 2-hydroxypyridine, 2-pyridone, 4-pyrimidone to have ${ }^{1} \pi \pi^{*}$ and ${ }^{1} n \pi^{*}$ character, respectively, in the FC region. However, this energetic ordering is reversed in the cases of 4-hydroxypyrimidine, 2,4-dihydroxypyrimidine, U and T. Fig. 7 illustrates the (expected) better spatial overlap between the orbitals involved in the $\pi^{*} \leftarrow \pi$ excitations $c f$. the $\pi^{*} \leftarrow \mathrm{n}$ excitations.

Several trends are evident in the data collected in Table 2. Amongst the enols, the ${ }^{1} \pi \pi^{*}-S_{0}$ term values are similar in phenol and 2-hydroxypyridine, and larger in 4-hydroxypyrimidine and 2,4-dihydroxypyrimidine. The similar term values in phenol and 2-hydroxypyridine can be understood if the weak intramolecular $\mathrm{H}$-bond between the $\mathrm{N}$ atom and the $\mathrm{OH}$ at the $\mathrm{C} 2$ position in the

Table 2 Calculated vertical and (where available) adiabatic excitation energies (VEEs and AEEs) for the first three excited singlet states of the pyrimidine systems, with the dominant transition character indicated in parenthesis next to the VEE values. Available experimental values are listed also, with (A) and (V) specifying whether the measured data should be compared with the calculated AEE or VEE values. The last two columns show, respectively, the optimised energy of any TS in the PEC along the LIIC linking the (vertical) ${ }^{1} \pi \pi^{\star}$ state and the ${ }^{1} \pi \pi^{\star} / \mathrm{S}_{0} \mathrm{MECl}$ and the energy of the ${ }^{1} \pi \pi^{\star} / \mathrm{S}_{0} \mathrm{MECl}$ itself. All energies are quoted in units of eV

\begin{tabular}{|c|c|c|c|c|c|c|c|c|}
\hline \multirow[b]{2}{*}{ Molecule ${ }^{\text {ref. }}$} & \multicolumn{3}{|l|}{$\Delta E\left(\mathrm{~S}_{1}-\mathrm{S}_{0}\right)$} & \multicolumn{2}{|l|}{$\underline{\Delta E\left(\mathrm{~S}_{2}-\mathrm{S}_{0}\right)}$} & \multirow{2}{*}{$\frac{\Delta E\left(\mathrm{~S}_{3}-\mathrm{S}_{0}\right)}{\mathrm{VEE}}$} & \multirow[t]{2}{*}{$\Delta E\left(\mathrm{TS}-\mathrm{S}_{0}\right)$} & \multirow[t]{2}{*}{$\Delta E\left(\mathrm{CI}-\mathrm{S}_{0}\right)$} \\
\hline & VEE & AEE & Exp. & VEE & Exp. & & & \\
\hline \multicolumn{9}{|l|}{ Enol } \\
\hline Phenol $^{9}$ & $4.57\left(\pi \pi^{*}\right)$ & 4.34 & $4.51(\mathrm{~A})$ & $5.40\left(\pi \sigma^{*}\right)$ & - & - & 4.89 & 4.63 \\
\hline 2-Hydroxy-pyridine ${ }^{154,155,157}$ & $4.70\left(\pi \pi^{*}\right)$ & 4.34 & $4.48(\mathrm{~A})$ & $6.17\left(\mathrm{n} \pi^{*}\right)$ & & $6.79\left(\mathrm{n} \sigma^{*}\right)$ & 4.71 & 4.32 \\
\hline 4-Hydroxy-pyrimidine & $4.94\left(\mathrm{n} \pi^{*}\right)$ & 4.64 & - & $5.18\left(\pi \pi^{*}\right)$ & - & $5.28\left(\mathrm{n} \pi^{*}\right)$ & 4.55 & 3.81 \\
\hline 2,4-Dihydroxy-pyrimidine & $4.90\left(\mathrm{n} \pi^{*}\right)$ & 4.52 & - & $5.02\left(\pi \pi^{*}\right)$ & - & $6.43\left(\pi \sigma^{*}\right)$ & - & 3.80 \\
\hline \multicolumn{9}{|l|}{ Keto } \\
\hline 2-Pyridone ${ }^{156,195-197}$ & $4.34\left(\pi \pi^{*}\right)$ & 3.57 & $3.70(\mathrm{~A})$ & $4.81\left(\mathrm{n} \pi^{*}\right)$ & - & $5.15\left(\mathrm{n} \sigma^{*}\right)$ & - & 3.70 \\
\hline 4-Pyrimidone & $4.45\left(\pi \pi^{*}\right)$ & - & 3.85 (A) & $4.92\left(\mathrm{n} \pi^{*}\right)$ & - & $5.58\left(\mathrm{n} \pi^{*}\right)$ & - & 3.12 \\
\hline Uracil $^{198}$ & $4.80\left(\mathrm{n} \pi^{*}\right)$ & 4.17 & - & $5.69\left(\pi \pi^{*}\right)$ & $5.08(\mathrm{~V})$ & $5.89\left(\pi \sigma^{*}\right)$ & - & 3.92 \\
\hline Thymine ${ }^{199}$ & $4.85\left(\mathrm{n} \pi^{*}\right)$ & 4.17 & - & $5.50\left(\pi \pi^{*}\right)$ & $4.80(\mathrm{~V})$ & $5.64\left(\pi \sigma^{*}\right)$ & - & 4.09 \\
\hline Cytosine $e^{60,119}$ & $4.58\left(\pi \pi^{*}\right)$ & - & - & $4.88\left(\mathrm{n} \pi^{*}\right)$ & 3.95 (A) & $5.34\left(\mathrm{n} \pi^{*}\right)$ & - & 3.46 \\
\hline
\end{tabular}




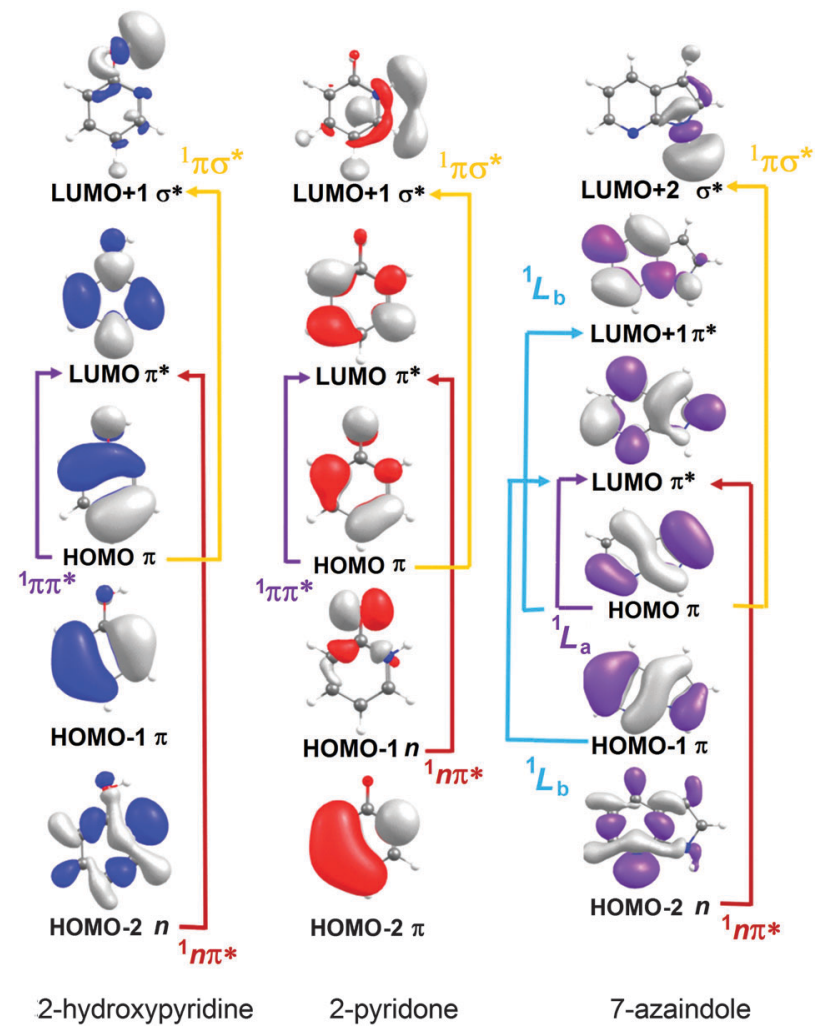

Fig. 7 Dominant orbital promotions involved in forming the first few singlet excited states of 2-hydroxypyridine, 2-pyridone and 7-azaindole.

latter provides a similar stabilisation to both the $\pi$ and $\pi^{*}$ orbitals. $\mathrm{OH}$ group(s) are $\pi$-donors. This additional electron density is preferentially localised at positions ortho- and para- to the ringatom bearing the $\mathrm{OH}$ group, and can be accommodated more readily by a more electronegative $\mathrm{N}$ atom ( $c f$. a $\mathrm{C}$ atom). The increased ${ }^{1} \pi \pi^{*}-\mathrm{S}_{0}$ term values in 4-hydroxypyrimidine and 2,4-dihydroxypyrimidine (wherein the ${ }^{1} \pi \pi^{*}$ state is raised above the $1^{1} \mathrm{n} \pi^{*}$ state) are thus attributed to preferential stabilisation of the $\pi$ (and destabilisation of the $\pi^{*}$ ) orbital by the additional $\mathrm{N}$ atom. Table 2 also shows that the $\mathrm{S}_{1}$ states of the keto tautomers (2-pyridone and 4-pyrimidone) are formed by the same orbital promotions as the corresponding enols, and that their ${ }^{1} \pi \pi^{*}-\mathrm{S}_{0}$ term values are consistently smaller, despite the gradual stabilisation of the $S_{0}$ state of the keto form upon $\mathrm{N}$ atom inclusion (recall Table 1 ). This can be understood by considering the differing $\pi$ donating capabilities of $\mathrm{NH}$ and $\mathrm{OH}$ groups, which will be amplified by the fact that the $\mathrm{OH}$ group is pendant to the ring. The in-ring $\mathrm{N}$ atom in the $\mathrm{NH}$ group is much more effective at stabilizing the electron deficiency introduced by $\pi^{*} \leftarrow \pi$ excitation (and thus the ${ }^{1} \pi \pi^{*}-\mathrm{S}_{0}$ energy gap) in the keto tautomers.

Photodissociation vs. photostability. As noted in the Introduction, photoinduced $\mathrm{O}-\mathrm{H}$ or $\mathrm{N}-\mathrm{H}$ bond fission is a recognised population loss process for many small, functionalised aromatic and heteroaromatic molecules, including phenols and azoles. ${ }^{1,2,7}$ In the case of phenol, near UV excitation to the $\mathrm{S}_{1}\left(1^{1} \pi \pi^{*}\right)$ state leads to $\mathrm{O}-\mathrm{H}$ bond fission, on a nanosecond timescale.


Fig. 8 Potential energy profiles for the ground and lowest singlet excited states along, respectively, $R_{\mathrm{X}-\mathrm{H}}(\mathrm{X}=\mathrm{O}, \mathrm{N})$ and $Q_{\mathrm{oop}}$ for phenol ((a) and (b)), 2-hydroxypyridine ((c) and (d)) and 2-pyridone ((e) and (f)). Filled points define the energies of the optimised critical structures (i.e. the $S_{0}$ and $1^{1} \pi \pi^{*}$ minima, the $\mathrm{S}_{0} /{ }^{1} \pi \pi^{\star} \mathrm{Cl}$ and the TS on the $1^{1} \pi \pi^{\star}$ potential leading to the prefulvenic $\mathrm{S}_{0} /{ }^{1} \pi \pi^{*} \mathrm{CI}$ ). The structures in each panel illustrate, respectively, the $\mathrm{X}-\mathrm{H}$ bond dissociation coordinate and the optimised geometry at the prefulvenic $\mathrm{S}_{0} /{ }^{1} \pi \pi^{\star} \mathrm{Cl}$.

Such behaviour can be understood in terms of the potential energy cuts (PECs) shown in Fig. 8(a). The ${ }^{1} \pi \pi^{*}$ state is diabatically bound with respect to stretching $R_{\mathrm{O}-\mathrm{H}}$, but the ${ }^{1} \pi \pi^{*}$ and ${ }^{1} \pi \sigma^{*}$ potentials experience a CI at slightly extended bond lengths $\left(R_{\mathrm{O}-\mathrm{H}} \sim 1.2 \AA\right)$, and $\mathrm{O}-\mathrm{H}$ bond fission can occur by tunnelling under this CI. ${ }^{16,17,26,27}$

The ns lifetime of the $1^{1} \pi \pi^{*}$ state of phenol implies the absence of any efficient rival non-radiative decay processes. One such non-radiative decay process identified in similar photoexcited molecules is internal conversion to the ground state facilitated by a CI between the $1^{1} \pi \pi^{*}$ and $\mathrm{S}_{0}$ states that can be accessed by motion along an out-of-plane ring deformation 
coordinate, $Q_{\text {oop }}$ (via a so-called prefulvenic geometry). ${ }^{14,149-152}$ Fig. 8(b) shows the calculated geometry of this $1^{1} \pi \pi^{*} / \mathrm{S}_{0}$ MECI in phenol - with $\mathrm{C} 1$ very obviously out of the plane. As in later figures of this type, the open points show the ground (and excited) state energies calculated at a sequence of points along the LIIC connecting the optimised $\mathrm{S}_{0}$ state geometry to that of the $1^{1} \pi \pi^{*} / S_{0}$ MECI. The small number of filled points show the energies of the optimised critical structures (notably the minimum of the $1^{1} \pi \pi^{*}$ state and the energy of any TS separating this minimum excited state geometry from the MECI) calculated at the CASPT2/AVDZ level of theory. These optimised energies are also listed in Table 2. In the specific case of phenol, the key finding from these calculations is the presence of a substantial $(\sim 0.5 \mathrm{eV})$ energy barrier in the MEP towards this prefulvenic MECI, that would be sufficient to render this pathway closed following excitation at energies not far above the origin of the $1^{1} \pi \pi^{*}$ state.

Fig. 8(c) and (d) show the corresponding PECs along $R_{\mathrm{O}-\mathrm{H}}$ and the prefulvenic $Q_{\text {oop }}$ coordinate for 2-hydroxypyridine. The most obvious feature of the former is the predicted elevation of the $1^{1} \pi \sigma^{*}$ potential (or, more precisely, stabilisation of the $S_{0}$ state by the intramolecular $\mathrm{H}$-bonding interaction between the $\mathrm{OH}$ group and the in-plane orbital on the adjacent ring $\mathrm{N}$ atom), which manifests itself as an increased barrier to tunnelling under the $1^{1} \pi \pi^{*} / 1^{1} \pi \sigma^{*}$ CI ( $c f$. phenol). Indeed, if the potentials along the LIIC displayed in Fig. 8(c) are anything like quantitative, this barrier would preclude $\mathrm{O}-\mathrm{H}$ bond fission as a possible decay process following excitation near the minimum of the $1^{1} \pi \pi^{*}$ state of 2-hydroxypyridine. As Fig. 8(d) shows, the predicted MECI along $Q_{\text {oop }}$ lies at an energy below that of the $1^{1} \pi \pi^{*}$ minimum but, as in phenol, motion towards this prefulvenic $1^{1} \pi \pi^{*} / \mathrm{S}_{0} \mathrm{CI}$ is impeded by an energy barrier (the calculated energy of the optimised TS is $\sim 0.37 \mathrm{eV}$ above that of the $1^{1} \pi \pi^{*}$ state minimum (see Table 2)). Once again, therefore, theory would predict a certain photophysical stability to the $1^{1} \pi \pi^{*}$ state of 2-hydroxypyridine, consistent with previous theoretical studies ${ }^{153,154}$ and experimental observations of well-resolved vibronic structure in the jet-cooled REMPI spectrum of this tautomer. ${ }^{155,156}$ We also note that geminate recombination at the $1^{1} \pi \pi^{*} / S_{0}$ CI could lead to branching into the $\mathrm{S}_{0}$ state of the keto tautomer via a photoinduced dissociation-association reaction. Though entropically unfavourable under isolated gas-phase conditions, such a process would certainly merit consideration if the chromophore was suspended in a raregas matrix or in bulk solution.

Analogous PECs for 2-pyridone are presented in Fig. 8(e) and (f). The profiles along the X-H stretch coordinate $\left(R_{\mathrm{N}-\mathrm{H}}\right.$ in this case) show qualitative similarities with those for the $1^{1} \pi \pi^{*}$ and $1^{1} \pi \sigma^{*}$ states of phenol (Fig. 8(a)) but the optimised energy of the $1^{1} \pi \pi^{*}$ state of 2-pyridone is well below the $\mathrm{N}-\mathrm{H}$ dissociation limit, thereby excluding $\mathrm{N}-\mathrm{H}$ bond fission as a possible decay channel following excitation at energies around the $1^{1} \pi \pi^{*}-S_{0}$ origin. The predicted MECI in the prefulvenic $Q_{\text {oop }}$ coordinate is below the $1^{1} \pi \pi^{*} \leftarrow \mathrm{S}_{0}$ vertical excitation energy, however (even after geometry optimisation, see Table 2), and the calculated PEC along the LIIC to the $1^{1} \pi \pi^{*} / \mathrm{S}_{0}$ CI is essentially barrierless. Such predictions also appear to be consistent with the available experimental data:
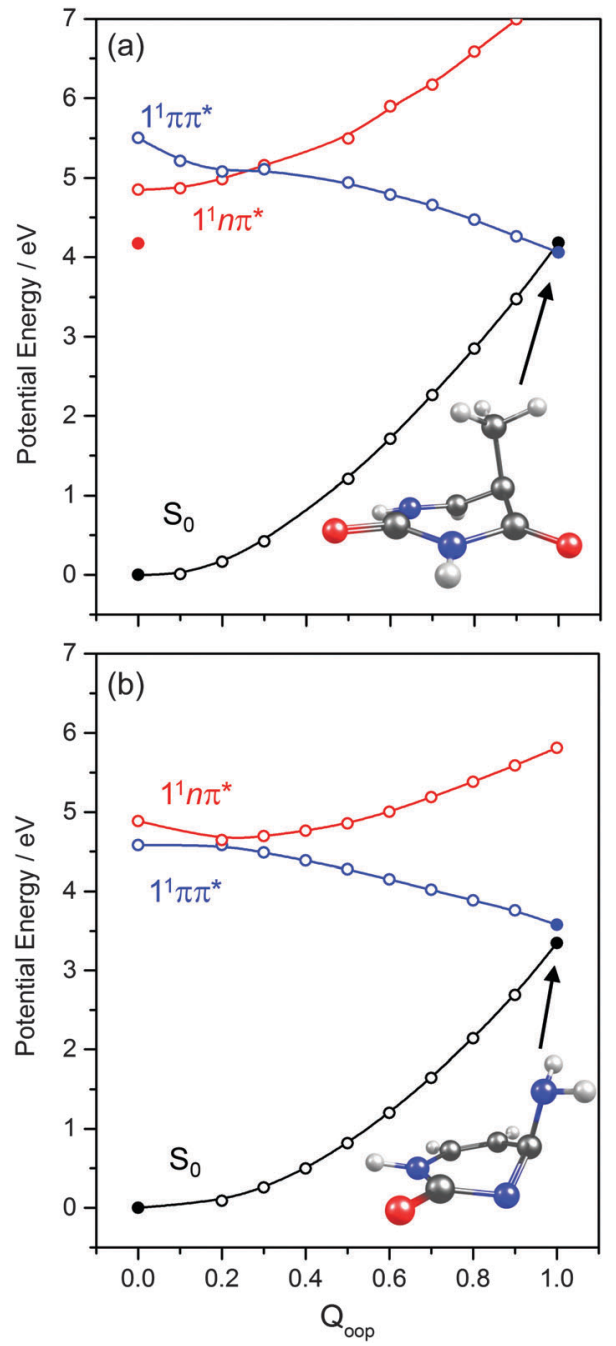

Fig. 9 Potential energy profiles along the out-of-plane ring deformation coordinate $Q_{\text {oop }}$ for the ground and first few singlet excited states of (a) $T$ and (b) $C$. Filled points represent the energies of the optimised critical structures (the $S_{0}$ and ${ }^{1} n \pi^{\star}$ state minima and the $S_{0} /{ }^{1} \pi \pi^{*} \mathrm{MECl}$ ) and the structure in each panel shows the optimised geometry of this $\mathrm{MECl}$.

the (split) origin band of the $1^{1} \pi \pi^{*}-\mathrm{S}_{0}$ transition of 2 -pyridone shows resolved rovibronic structure and the dispersed emission spectrum following excitation to the origin level(s) reveals population of an extensive range of ground state vibrational levels. ${ }^{157}$ The latter observation implies that the $1^{1} \pi \pi^{*}$ and $S_{0}$ states of 2-pyridone have different equilibrium geometries (as is also implied by the difference in the VEE and AEE values reported in Table 2). The reported two colour $1+1^{\prime}$ REMPI spectrum via the $1^{1} \pi \pi^{*}$ state, in contrast, is dominated by the origin band(s) - a finding that can most readily be explained by invoking a sharp drop in excited state lifetime upon tuning to higher energies. ${ }^{154,155,158}$

PECs along $Q_{\text {oop }}$ for the ground and first three excited singlet states of (a) thymine and (b) cytosine are shown in Fig. 9. Again, these energies were calculated at the CASPT2 level for a sequence of geometries along a LIIC linking the CASSCF optimised $\mathrm{S}_{0}$ minimum and the $\mathrm{S}_{0} / 1^{1} \pi \pi^{*}$ MECI. These values are also reported in Table 2 . The similarities between these 
PECs for $\mathrm{T}$ and $\mathrm{C}$, and those for 2-pyridone (Fig. 8(f)), are obvious, notwithstanding the reversal in the energetic ordering of the $1^{1} \mathrm{n} \pi$ and $1^{1} \pi \pi^{*}$ states. In both $\mathrm{T}$ and $\mathrm{C}$, the calculated $1^{1} \pi \pi^{*}$ PEC leading to the $1^{1} \pi \pi^{*} / \mathrm{S}_{0}$ MECI in the $Q_{\text {oop }}$ coordinate is barrierless. Indeed, the predicted energy of the $1^{1} \pi \pi^{*} / \mathrm{S}_{0}$ MECI is even below that of the $1^{1} n \pi$ origin - consistent with previous observations of ultrafast excited state decay following UV excitation of both $\mathrm{T}$ and C. ${ }^{54,55,57,59}$

Notwithstanding the rich literature addressing the excited state photophysics of the DNA/RNA bases, there have been few systematic studies of the electronic and geometric reasons why such out-of-plane ring deformations are effective in promoting ultrafast excited state decay - and thus photostability - in pyrimidine derivatives like $\mathrm{T}$ and $\mathrm{C}$, but not in simpler analogues like phenol. Yet recognising the factors that underpin this photostability could be important in guiding the development of more efficient photoprotective molecules for use in, e.g., sunscreens or artificial intraocular lenses. Thus we now summarise, and seek to rationalise, how the introduction of $\mathrm{N}$ atoms into the phenol ring and the addition of further $\mathrm{OH}$ and/or $\mathrm{NH}_{2}$ functionalities influences the topography of the excited state PES(s) along $Q_{\text {oop }}$ en route to the MECI with prefulvenic geometry.

Trends with respect to out-of-plane deformation. To aid this discussion, Fig. 10 presents an overlay of the calculated $1^{1} \pi \pi^{*}$ excited state PECs for phenol, 2-hydroxypyridine, 4-hydroxypyrimidine and 2,4-dihydroxypyrimidine (i.e. the enol tautomers listed in Table 2) plotted as a function of the out-of-plane deformation coordinate leading to the optimised $1^{1} \pi \pi^{*} / \mathrm{S}_{0}$ MECI. A similar overlay for the corresponding keto tautomers is shown in Fig. 11. The energies associated with each $1^{1} \pi \pi^{*}$ PEC are referenced to the minimum energy of the respective $S_{0}$ states but, for clarity and because the $\mathrm{S}_{0}$ PECs in $Q_{\text {oop }}$ are all rather similar, only one $\mathrm{S}_{0}$ PEC (for 2-hydroxypyridine in Fig. 10, and for 2-pyridone in Fig. 11) is displayed in each panel. More detailed depictions of the ground and excited state PECs for these molecules, and side-on views illustrating the out-of-plane displacements of the ring atoms at the various $S_{0} / 1^{1} \pi \pi^{*}$ MECIs are provided in Fig. S1-S9 in the ESI. $\dagger$ The optimised energies of the various $1^{1} \pi \pi^{*} / \mathrm{S}_{0}$ MECIs $\left(\Delta E\left(\mathrm{CI}-\mathrm{S}_{0}\right)\right)$ and of any $\mathrm{TS}$ in the PEC along the LIIC linking the (vertical) $1^{1} \pi \pi^{*}$ state and the $1^{1} \pi \pi^{*} / \mathrm{S}_{0}$ MECI $\left(\Delta E\left(\mathrm{TS}-\mathrm{S}_{0}\right)\right)$ are included in Table 2.

Inspecting these data, we see that the VEEs of the respective $1^{1} \pi \pi^{*}$ states increase across the enol series phenol $\rightarrow$ 2,4-dihydroxypyrimidine, while the energies of the $1^{1} \pi \pi^{*} / S_{0}$ MECIs decrease. As a consequence, the barrier to accessing the $1^{1} \pi \pi^{*} / \mathrm{S}_{0}$ MECI decreases across the series and the gradient along $Q_{\text {oop }}$ becomes progressively steeper with increasing $\mathrm{N}$ incorporation and ring functionalization. The VEEs of the $1^{1} \pi \pi^{*}$ states of the keto tautomers also increase across the series 2-pyridone $\rightarrow \mathrm{U}$ and $\mathrm{T}^{159}$ The energies of the $1^{1} \pi \pi^{*} / \mathrm{S}_{0}$ MECIs increase in much the same order, but are always below the corresponding VEEs. The $1^{1} \pi \pi^{*}$ PECs leading to these MECIs have similar gradients in all four species, and none shows any obvious barrier. This difference in topography (cf. the enol tautomers) can plausibly be traced to differences in the extent of the $\pi$-conjugation in the enol and keto families.

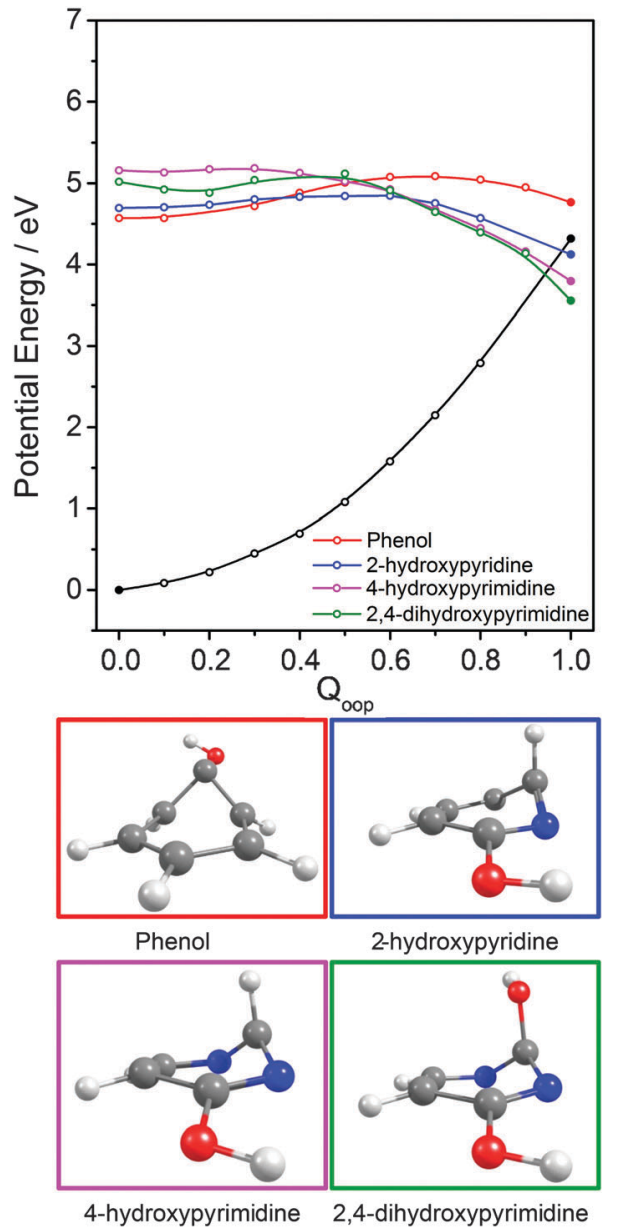

Fig. 10 Overlaid PECs along $Q_{\text {oop }}$ for the ${ }^{1} \pi \pi^{\star}$ states and respective ${ }^{1} \pi \pi^{\star} / S_{0} \mathrm{MECls}$ for phenol, 2-hydroxypyridine, 4-hydroxypyrimidine and 2,4-dihydroxypyrimidine along with the calculated $S_{0}$ PEC for 2-hydroxypyridine. The filled points show the energies of the optimised critical structures (i.e. the $\mathrm{S}_{0}$ state and the $\mathrm{S}_{0} /{ }^{1} \pi \pi^{\star} \mathrm{Cl}$ ). The optimised geometry at each $\mathrm{MECl}$ is shown below.

The $\mathrm{sp}^{2}$ hybridisation of the $\mathrm{N}$ atom in 2-hydroxypyridine, for example, would be expected to be stronger than that in the keto analogue (2-pyridone), and thus more resistant to initial out of plane deformation - consistent with the barrier predicted in the PEC along $Q_{\text {oop }}$ in the former.

Various trends revealed in these data are now considered in turn. The ground state of each of these systems is aromatic, the equilibrium geometry of the ring atoms is planar and the potential energy increases with out-of-plane ring deformation. Introducing one or more heteroatoms into the ring will weaken the rigidity, whereas adding $\mathrm{OH}$ groups (as in the enols) and, particularly, $\mathrm{C}=\mathrm{O}$ groups (as in the keto tautomers) tends to reinforce the preference for planarity by extending the $\pi$-conjugation. $\pi^{*} \leftarrow \pi$ excitation destroys the aromaticity, induces some expansion of the ring, and lowers the resistance to out-of-plane ring deformation - to the extent that, in all bar phenol, the $1^{1} \pi \pi^{*}$ state potential is either essentially flat, or unstable, with respect to out-of-plane deformation. Under such circumstances, a CI with the $\mathrm{S}_{0}$ PES upon distorting along 

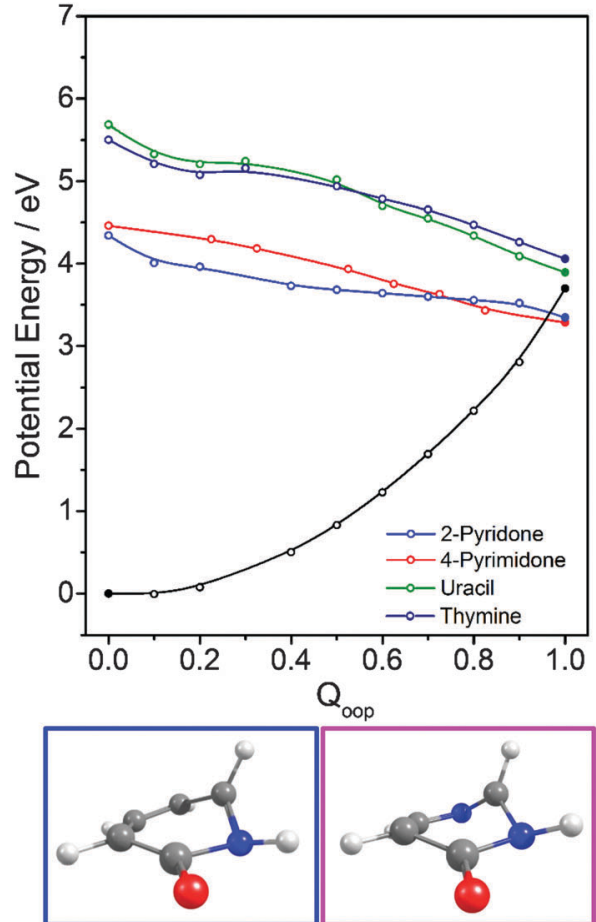

2-pyridone 4pyrimidone

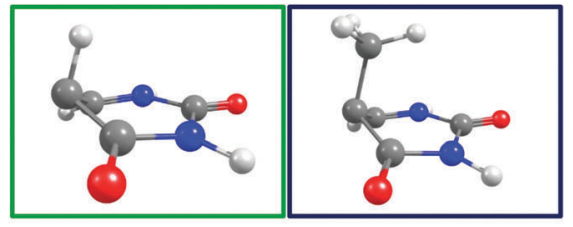

Uracil

Thymine

Fig. 11 Overlaid PECs along $Q_{\text {oop }}$ for the ${ }^{1} \pi \pi^{\star}$ states and respective ${ }^{1} \pi \pi^{\star} / S_{0}$ MECls for 2-pyridone, 4-pyrimidone, $U$ and $T$ along with the calculated $S_{0}$ PEC for 2 -pyridone. The filled points show the energies of the optimised critical structures (i.e. the $\mathrm{S}_{0}$ state and the $\mathrm{S}_{0} /{ }^{1} \pi \pi^{\star} \mathrm{Cl}$ ). The optimised geometry at each $\mathrm{MECl}$ is shown below.

$Q_{\text {oop }}$ is inevitable. As Fig. 10 and Table 2 show, the energy of the $1^{1} \pi \pi^{*} / \mathrm{S}_{0}$ MECI in $Q_{\text {oop }}$ decreases upon introducing one or more $\mathrm{N}$ atoms into the ring. In all cases other than phenol, the $1^{1} \pi \pi^{*} / \mathrm{S}_{0}$ MECI in $Q_{\text {oop }}$ is calculated to lie below the VEE of the $1^{1} \pi \pi^{*}$ (and the $1^{1} n \pi^{*}$ ) excited states. These MECIs also lie below the calculated $\mathrm{O}-\mathrm{H}$ and $\mathrm{N}-\mathrm{H}$ bond dissociation energies in 2-hydroxypyridine and 2-pyridone. Further, recalling Fig. 8(c) and (e), the latter excited state bond fissions are predicted to be hampered by a potential barrier (the lower part of the $1^{1} \pi \pi^{*} / 1^{1} \pi \sigma^{*}$ CI in $\left.R_{\mathrm{X}-\mathrm{H}}(\mathrm{X}=\mathrm{N}, \mathrm{O})\right)$. Thus we conclude that, at least relative to $\mathrm{X}-\mathrm{H}$ bond fission, IC to the $\mathrm{S}_{0}$ state mediated by out-of-plane distortion is likely to be the dominant decay process following long wavelength excitation of the N-containing heterocycles in Table 2 . However, we also recognise that the excited state decay rate will be sensitive not just to the detailed topography of the excited state PES connecting the FC region to the $1^{1} \pi \pi^{*} / \mathrm{S}_{0}$ MECI in $Q_{\text {oop }}$ (which may be influenced by substitution as, for example, for the cases of 5 -hydroxy- and 5 -aminouracil ${ }^{160}$ ), but also to the excitation energy.
The detailed form of the out-of-plane displacement leading to the $1^{1} \pi \pi^{*} / \mathrm{S}_{0}$ MECI is also molecule dependent. As noted above, $\pi^{*} \leftarrow \pi$ excitation results in some 'loosening' of the ring structure and localisation of the $\pi$-bonding, with one or more ring atoms tending towards a pyramidal bonding arrangement. Among the enols, the atom exhibiting the greatest out-of-plane distortion (defined relative to the best-fit plane through the six ring atoms at the calculated geometry of the $1^{1} \pi \pi^{*} / \mathrm{S}_{0}$ MECI) varies between $\mathrm{C} 1$ in phenol, C6 in 2-hydroxypyridine and C2 in 4-hydroxypyrimidine and 2,4-dihydroxypyrimidine - as shown in Fig. 10 and Fig. S1-S4 (ESI $\dagger$ ). The $1^{1} \pi \pi^{*} / S_{0}$ MECI identified in phenol has a classic prefulvenic structure, with $\mathrm{C} 1$ (i.e. the $\mathrm{C}$ atom supporting the $\mathrm{OH}$ group) showing the greatest out-ofplane distortion, while the finding that the $\mathrm{C} 2$ atom in both pyrimidines shows the greatest out-of-plane distortion reflects its location between the two $\mathrm{N}$ atoms, which more readily adopt a local $\mathrm{sp}^{3}$ (pyramidal) coordination. The carbonyl group(s) in the keto tautomers encourage local planarity; relative to 2,4-dihydroxypyrimidine, $\mathrm{C} 2$ in $\mathrm{U}$ (and $\mathrm{T}$ ) is less distorted out of the plane, with strain relief provided, instead, by increased puckering at C5 and C6.

The VEEs of the $1^{1} n \pi^{*}$ states of 4-hydroxypyrimidine, 2,4-dihydroxypyrimidine, $\mathrm{U}$ and $\mathrm{T}$ are all calculated to be below those of the corresponding $1^{1} \pi \pi^{*}$ states (see Table 2) and, as Fig. 9 showed (for the specific cases of $\mathrm{T}$ ), the respective PESs show a CI in the $Q_{\text {oop }}$ coordinate. As suggested previously, ${ }^{78-81,86,87,91,94,103}$ IC to the $1^{1} \mathrm{n} \pi^{*}$ state could thus constitute another possible non-radiative decay pathway following excitation to the 'bright' $1^{1} \pi \pi^{*}$ state in these molecules. Conversely, one might also envisage instances where IC to the $1^{1} \pi \pi^{*}$ state (and subsequent coupling to $S_{0}$ via the $1^{1} \pi \pi^{*} / S_{0}$ MECI) could provide a non-radiative population loss route following excitation to higher vibrational levels within the $1^{1} \mathrm{n} \pi^{*}$ manifold.

\subsection{Bicyclic ring systems and the purine bases}

Calculated VEEs for the first three excited singlet states of each molecule shown in Fig. 2(a)-(f) are listed in Table 3, along with the dominant character of the various excitations (in parenthesis). The excited states in each case comprise two ${ }^{1} \pi \pi^{*}$ states, traditionally labelled ${ }^{1} \mathrm{~L}_{\mathrm{a}}$ and ${ }^{1} \mathrm{~L}_{\mathrm{b}}$, and a ${ }^{1} \mathrm{n} \pi^{*}$ state. Apart from in the case of indole, the lowest lying ${ }^{1} \pi \sigma^{*}$ excited state has not been included in the present calculations, but $\mathrm{H}$ atom photofragment translational spectroscopy measurements following near UV photolysis of gas phase indole $e^{33}$ and adenine ${ }^{161}$ molecules suggest that the threshold energy for $\mathrm{N}-\mathrm{H}$ bond fission following radiationless transfer to the $1^{1} \pi \sigma^{*}$ state in these molecules is $\sim 4.71 \mathrm{eV}$ and $\sim 5.33 \mathrm{eV}$, respectively.

The dominant orbital promotions involved in forming these excited states in 7-azaindole were shown in Fig. 7. The ${ }^{1} \mathrm{~L}_{\mathrm{a}}$ and ${ }^{1} \mathrm{~L}_{\mathrm{b}}$ states differ in the detail of the participating $\pi$ and $\pi^{*}$ orbitals. The ${ }^{1} \mathrm{~L}_{\mathrm{a}} \leftarrow \mathrm{S}_{0}$ transitions are generally pictured as $\pi^{*}($ LUMO $) \leftarrow \pi$ (HOMO) excitations and have the larger absorption cross-section. ${ }^{162}$ As in the pyrimidine systems, however, the energetic ordering of the ${ }^{1} \pi \pi^{*}\left({ }^{1} L_{a}\right.$ and $\left.{ }^{1} L_{b}\right)$ and ${ }^{1} n \pi *$ states is sensitive to $\mathrm{N}$ substitution (as Table 3 shows, the $1^{1} n \pi^{*}$ state 
Table 3 Calculated VEEs and (where available) AEEs for the first three singlet excited states of the purine systems, with the dominant character of the transition indicated in parenthesis next to the VEE values. Available experimental values are listed also, with (A) and (V) specifying whether the measured data should be compared with the calculated AEE or VEE values. The last two columns show, respectively, the (unrelaxed) energy of any TS in the PEC along the LIIC linking the (vertical) ${ }^{1} \pi \pi^{*}$ state and the ${ }^{1} \pi \pi^{*} / \mathrm{S}_{0} \mathrm{MECl}$, and the optimised energy of the ${ }^{1} \pi \pi^{\star} / \mathrm{S}_{0} \mathrm{MECl}$. All energies are quoted in units of eV

\begin{tabular}{|c|c|c|c|c|c|c|c|c|}
\hline Molecule $^{\text {ref. }}$ & \multicolumn{3}{|l|}{$\Delta E\left(\mathrm{~S}_{1}-\mathrm{S}_{0}\right)$} & \multicolumn{2}{|l|}{$\Delta E\left(\mathrm{~S}_{2}-\mathrm{S}_{0}\right)$} & $\frac{\Delta E\left(\mathrm{~S}_{3}-\mathrm{S}_{0}\right)}{\mathrm{VEE}}$ & $\Delta E\left(\mathrm{TS}-\mathrm{S}_{0}\right)$ & $\Delta E\left(\mathrm{CI}-\mathrm{S}_{0}\right)$ \\
\hline Indole ${ }^{33,200}$ & $4.55\left(\pi \pi^{*}\right)$ & 4.44 & $4.37(\mathrm{~A})$ & $5.07\left(\pi \pi^{*}\right)$ & $4.54(\mathrm{~A})$ & $6.82\left(\pi \sigma^{*}\right)$ & 5.17 & 5.06 \\
\hline 5,7-Azaindole & $4.50\left(\mathrm{n} \pi^{*}\right)$ & & - & $4.97\left(\pi \pi^{*}\right)$ & - & $5.11\left(\pi \pi^{*}\right)$ & - & 3.82 \\
\hline Purine $e^{205}$ & $4.70\left(\mathrm{n} \pi^{*}\right)$ & 4.59 & 3.88 (A) & $4.99\left(\pi \pi^{*}\right)$ & - & $5.68\left(\pi \pi^{*}\right)$ & - & 4.01 \\
\hline Adenine $^{160-206}$ & $4.86\left(\mathrm{n} \pi^{*}\right)$ & & $4.40(\mathrm{~A})$ & $5.13\left(\pi \pi^{*}\right)$ & 4.48 (A) & $5.28\left(\pi \pi^{*}\right)$ & - & 3.90 \\
\hline Guanine $^{207}$ & $5.25\left(\pi \pi^{*}\right)$ & - & $4.13(\mathrm{~A})$ & $5.55\left(\mathrm{n} \pi^{*}\right)^{a}$ & - & $6.53\left(\mathrm{n} \pi^{*}\right)$ & - & 3.47 \\
\hline
\end{tabular}
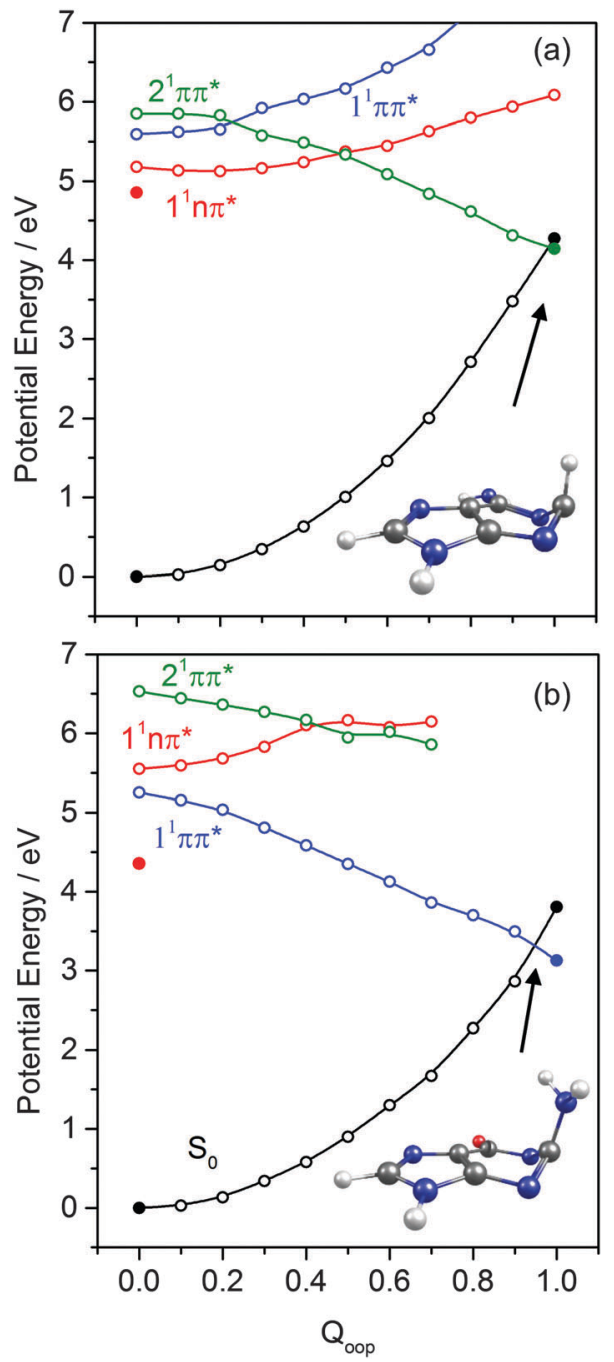

Fig. 12 Diabatic PECs along $Q_{\text {oop }}$ for the ground and first three singlet excited states of (a) A and (b) G. The filled points depict the energies of optimised critical structures (i.e. the $S_{0}$, and $1^{1} n \pi^{\star}$ state minimum energy geometries, and the $\mathrm{S}_{0} /^{1} \pi \pi^{\star} \mathrm{MECl}$ - the optimised geometry of which is also shown).

is the $S_{1}$ state in 5,7-azaindole, purine and A) and can be modified by solvation. ${ }^{163}$ The relative excited state energies are also sensitive functions of $Q_{\mathrm{oop}}$, as shown for the cases of A and G in Fig. 12.
Viewed in terms of these diabatic PECs in $Q_{\text {oop }}$, we find that the higher energy $2^{1} \pi \pi^{*}\left({ }^{1} L_{a}\right)$ PEC correlates with the ${ }^{1} \pi \pi^{*} / S_{0}$ MECI in 5,7-azaindole, purine, A and G, whereas in indole and 7-azaindole it is the $1^{1} \pi \pi^{*}\left({ }^{1} \mathrm{~L}_{\mathrm{b}}\right)$ PEC that correlates with this MECI. Fig. 13 compares calculated PECs along the LIIC linking the relevant (vertical) ${ }^{1} \pi \pi^{*}$ excited state and the ${ }^{1} \pi \pi^{*} / \mathrm{S}_{0}$ MECI for indole, 7-azaindole, 5,7-azaindole, purine and A. Each is referenced to a common zero of energy (the respective $S_{0}$ minimum) but again, to avoid congestion, only the $\mathrm{S}_{0}$ PEC for indole is shown. More detailed depictions of the ground and excited state PECs for these molecules, and cross-sectional views showing the out-of-plane displacements of the ring atoms at each of the ${ }^{1} \pi \pi^{*} / \mathrm{S}_{0}$ MECIs are provided in Fig. S10-S15 in the ESI. $\dagger$ The optimised energies of the various ${ }^{1} \pi \pi^{*} / \mathrm{S}_{0}$ MECIs $\left(\Delta E\left(\mathrm{CI}-\mathrm{S}_{0}\right)\right)$ and of any TS identified in this PEC along $Q_{\text {oop }}$ $\left(\Delta E\left(\mathrm{TS}-\mathrm{S}_{0}\right)\right)$ are included in Table 3.

Many of the trends evident in Fig. 13 are reminiscent of those discussed previously for the unicyclic systems. The ${ }^{1} \pi \pi^{*} /$ $\mathrm{S}_{0}$ MECI in indole is calculated to lie above the AEE of the $1^{1} \pi \pi^{*}$ state. Motion along $Q_{\text {oop }}$ is thus unlikely to promote IC following photoexcitation at the $1^{1} \pi \pi^{*}\left({ }^{1} L_{b}\right) \leftarrow S_{0}$ origin consistent with the reported $(\sim 17 \mathrm{~ns})$ excited state lifetime. ${ }^{164,165}$ As Fig. 13 shows, however, the introduction of even one $\mathrm{N}$ atom into the six-membered ring raises the VEE of the ${ }^{1} \pi \pi^{*}$ state and lowers the energy of the ${ }^{1} \pi \pi^{*} / S_{0}$ MECI sufficiently that the PEC linking the FC region to the ${ }^{1} \pi \pi^{*} / \mathrm{S}_{0}$ MECI in $Q_{\text {oop }}$ becomes essentially barrierless. Notwithstanding that in most cases it is the diabatic $2^{1} \pi \pi^{*}$ PEC that links to this MECI, the available literature ${ }^{166}$ supports the expectation that molecules excited to (vibrationally excited levels of) the $1^{1} \pi \pi^{*}$ and $1^{1} n \pi^{*}$ states will find efficient radiationless coupling pathways to this (and related) CIs with the $\mathrm{S}_{0}$ PES.

The out-of-plane displacements required to access the various ${ }^{1} \pi \pi^{*} / \mathrm{S}_{0}$ MECIs also have obvious parallels with those discussed in Section 3.2. Again, we choose to define these displacements with respect to the plane of best-fit through the (nine in this case) ring atoms. As Fig. 13 and Fig. S10-S15 (ESI $\dagger$ ) show, the distortions are largely concentrated in the 6-membered ring. The atom showing the largest out-of-plane displacement at the ${ }^{1} \pi \pi^{*} / \mathrm{S}_{0}$ MECI is again molecule dependent, but the largest distortions are always remote from the bond that fuses the two ring systems. C5 is most displaced in indole, but this switches to $\mathrm{C6}$ in 7-azaindole 

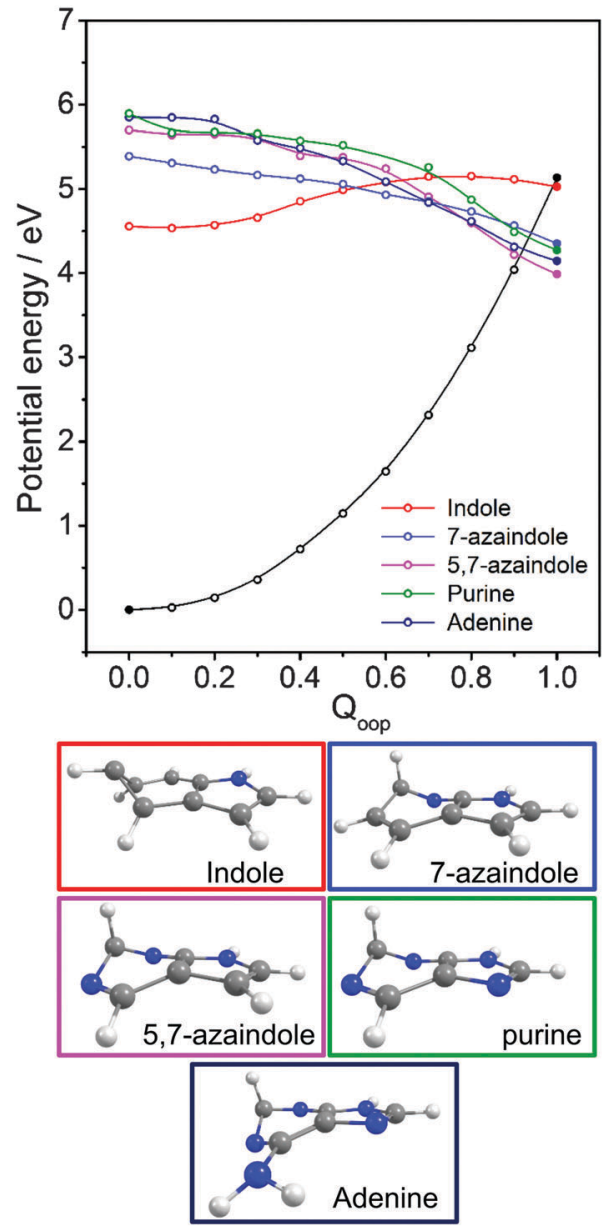

Fig. 13 Overlaid diabatic PECs along $Q_{\text {oop }}$ for the ${ }^{1} \pi \pi^{\star}$ state that correlates with the ${ }^{1} \pi \pi^{\star} / \mathrm{S}_{0} \mathrm{MECl}$ in indole, 7-azaindole, 5,7-azaindole, purine and $A$. The filled points show the energies of the optimised critical structures (i.e. the $\mathrm{S}_{0}$ state minimum and the $\mathrm{S}_{0} /{ }^{1} \pi \pi^{\star} \mathrm{MECl}$ ) and, for clarity, only the $S_{0}$ PEC calculated for indole is shown (open black points). The optimised geometry at each $\mathrm{MECl}$ is shown below.

(reflecting the energetic preference for $\mathrm{sp}^{3}$ hybridising the neighbouring $\mathrm{N} 7$ atom). The ${ }^{1} \pi \pi^{*} / \mathrm{S}_{0}$ MECI in 5,7-azaindole is characterised by an obvious puckering around N5 and C6. Introducing an additional $\mathrm{N}$ atom in the 5-membered ring (as in purine, $A$ and $G$ ) leads to a switch in the numbering convention used when describing the imidazole containing bicyclic systems - recall Fig. 2. It also allows greater relaxation of the bond common to the two rings (the $\mathrm{C} 4-\mathrm{C} 5$ bond in these molecules), with the result that C2 located between the N1 and N3 atoms is predicted to show the largest out-of-plane displacement at the ${ }^{1} \pi \pi^{*} / S_{0}$ MECI - as in the unicyclic analogues 4-hydroxypyrimidine, 2,4-dihydroxypyrimidine and 4-pyrimidone.

\subsection{The ribonucleosides and deoxyribonucleosides}

We now turn to the individual RNA base nucleosides: 5-methyluridine (uridine, thymidine), cytidine, adenosine and guanosine. The present study is limited to the oxygenated nucleosides with a ribose sugar attached to the $\mathrm{N}$ terminus of each base, but replacing
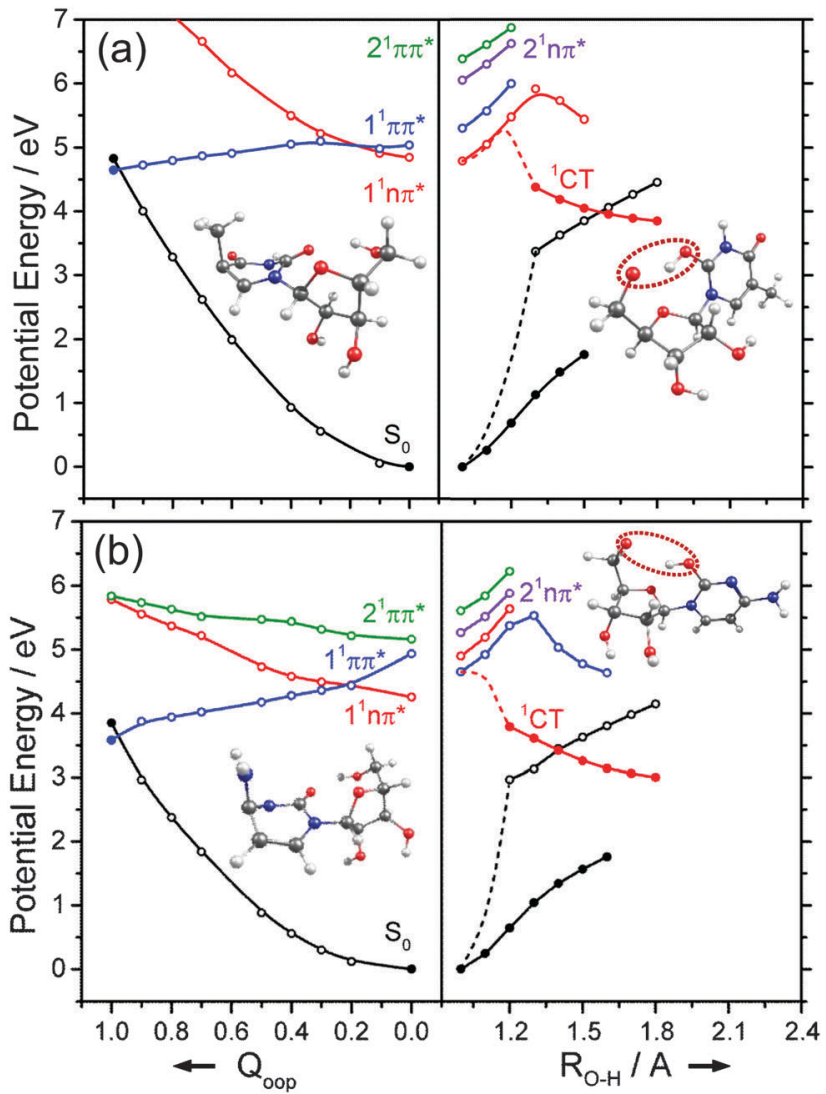

Fig. 14 Diabatic PECs for the ground and first few singlet excited states of (a) 5-methyluridine and (b) cytidine, along $Q_{\text {oop }}$ (left panel) and along the EDPT coordinate (right panel) calculated at CASPT2 and ADC(2) levels of theory, respectively. Filled points in the left hand panels represent the energies of the optimised critical structures (i.e. the $\mathrm{S}_{0}$ state minimum and the $\mathrm{S}_{0} /{ }^{1} \pi \pi^{\star} \mathrm{MECl}$ - the optimised geometry of which is also shown) whereas the convention for using open and filled points in the right hand panels is detailed in the text.

one $\mathrm{OH}$ group on the ribose sugar by an $\mathrm{H}$ atom (as in the deoxyribonucleosides) is not expected to have any substantial effect on the excited state photophysics described in this section. The CASPT2 calculated PECs along $Q_{\text {oop }}$ displayed in Fig. 14 and 15 exhibit obvious similarities with those of the precursor base. For comparative purposes, we have also scanned the $Q_{\text {oop }}$ coordinate of adenosine using the $\mathrm{ADC}(2) / \mathrm{cc}-\mathrm{pVDZ}$ level of theory. The resulting PECs, shown in Fig. S34 of the ESI, $\dagger$ are reassuringly similar to those calculated at the CASPT2/cc-pVDZ level.

The present calculations suggest that the $\mathrm{S}_{1}$ states of adenosine, 5-methyluridine and cytidine have predominant ${ }^{1} n \pi^{*}$ character in the FC region, whereas that of guanosine is largely of ${ }^{1} \pi \pi^{*}$ character. In each case we identify a ${ }^{1} \pi \pi^{*} / S_{0}$ MECI with prefulvenic geometry lying at an energy below the calculated $S_{1}$ state minimum. The calculated ${ }^{1} \pi \pi^{*}$ PEC from the FC region to this MECI is in all cases barrierless. Such findings reinforce the view that the nuclear deformations required to sample this MECI are base-centred and suggest that the oxygenated ribose sugar is largely a spectator in this particular radiationless pathway. More detailed inspection reveals that the energies of the various excited states and the prefulvenic ${ }^{1} \pi \pi^{*} / \mathrm{S}_{0}$ MECIs in 

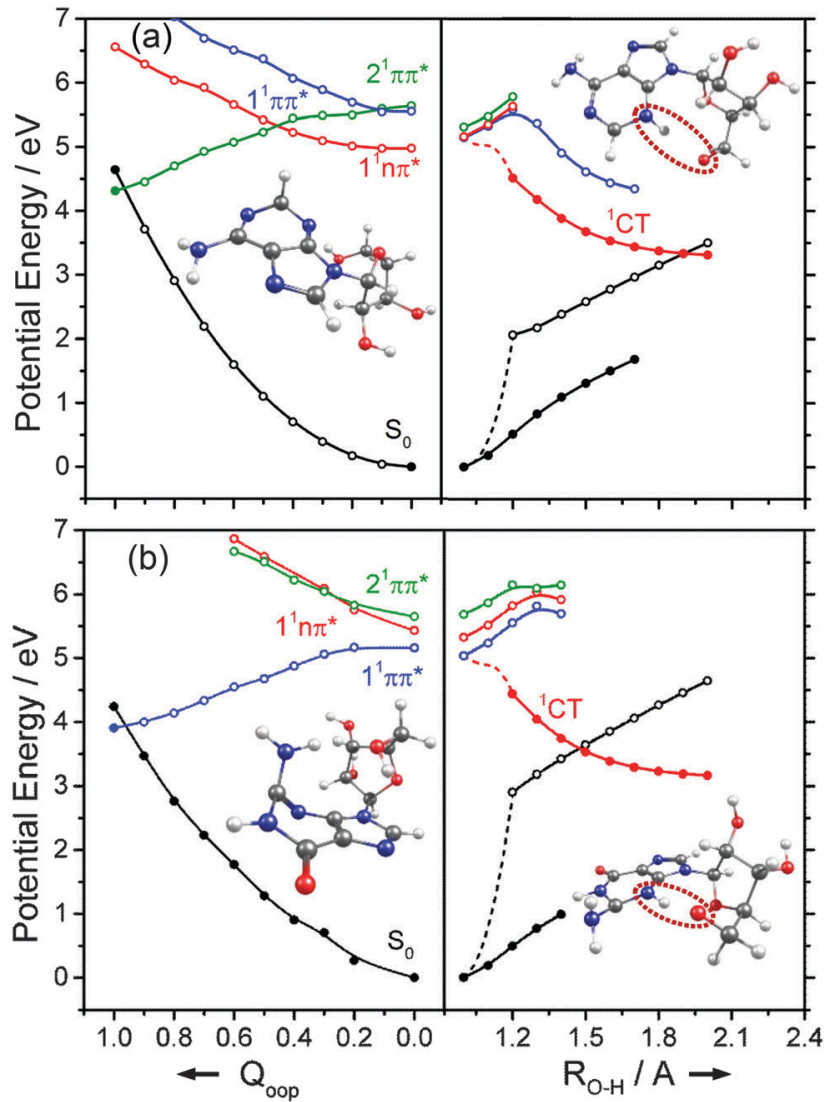

Fig. 15 Diabatic PECs for the ground and first three singlet excited states of (a) adenosine and (b) guanosine along $Q_{\text {oop }}$ (left panel) and along the EDPT coordinate (right panel) calculated at CASPT2 and ADC(2) levels of theory, respectively. Filled points in the left hand panels represent the energies of the optimised critical structures (i.e. the $S_{0}$ state minimum and the $S_{0} /{ }^{1} \pi \pi^{\star} \mathrm{MECl}$ - the optimised geometry of which is also shown). The convention for the use of open and filled points in the right hand panels is detailed in the text.

the nucleosides are slightly destabilised relative to the corresponding quantities in the isolated base. These relative increases in excitation energy can be traced to the stabilising effects of hydrogen bonding between an $\mathrm{O}-\mathrm{H}$ group of the ribose sugar and the base centred $\mathrm{N}$ or $\mathrm{O}$ atom in the ground state nucleoside.

Such H-bonding interactions are potentially important in the present context, as they can promote rival radiationless decay pathways in the DNA base residues, wherein the photoexcited state of interest develops charge-transfer (CT) character and undergoes intramolecular electron-driven proton (hydrogen) transfer (EDPT) from the terminal $\mathrm{O}-\mathrm{H}$ group of the ribose to an $\mathrm{N}$ or $\mathrm{O}$ atom in the aromatic ring. In contrast to the PECs along $Q_{\text {oop }}$ - which were calculated at the CASPT2 level to afford fairest comparison with the corresponding PECs for the smaller systems reported in Sections 3.1-3.3 - the PECs along the EDPT coordinate shown in the right hand panels of Fig. 14 and 15 were calculated at the ADC2 level. This is a pragmatic choice. The electronic states that promote EDPT involve orbital promotions that are sensitive to small changes in nuclear geometry. As such, a poorly chosen active space in CASSCF/CASPT2 can lead to spurious results along the charge-transfer coordinate, usually associated with important orbitals entering/leaving the active space. One way to circumvent this is to increase the active space substantially, but this leads to a large increase in computational expense. Given the available computational resources and (relatively) large size of the nucleoside molecules, the increased computational demands would have precluded detailed mechanistic study along the EDPT path with CASPT2 - especially in mapping the relaxed excited state paths.

The PECs along the EDPT coordinate were derived as follows: the $\mathrm{S}_{0}$ state was first scanned using the sugar $\mathrm{O}-\mathrm{H}$ bond as the driving coordinate (henceforth identified simply as $R_{\mathrm{O}-\mathrm{H}}$ ). The filled black points in each panel depict the 'relaxed' $S_{0}$ state energies calculated by fixing $R_{\mathrm{O}-\mathrm{H}}$ at selected values and allowing all other internal degrees of freedom to relax to their minimum energy configuration. The open coloured points show the various excited state energies calculated at the corresponding $\mathrm{S}_{0}$ state geometries. A relaxed scan was then constructed for the ${ }^{1} \mathrm{CT}$ state (filled red points), and the corresponding $S_{0}$ state energies calculated at the relaxed $\mathrm{S}_{1}$ geometries (shown by the open black symbols). The dashed black and red curves show the potential energy profiles for, respectively, the ground and first (adiabatic) excited states along the LIIC connecting the $\mathrm{S}_{0}$ minimum geometry and the relaxed ${ }^{1} \mathrm{CT}$ structure at the shortest $R_{\mathrm{O}-\mathrm{H}}$ distance. In all cases, the energy along the $\mathrm{S}_{0}$ optimised reaction path rises while that of the ${ }^{1} \mathrm{CT}$ state declines with increasing $R_{\mathrm{O}-\mathrm{H}}$, with the result that the two states become degenerate at extended $\mathrm{O}-\mathrm{H}$ bond lengths. The resulting ${ }^{1} \mathrm{CT} / \mathrm{S}_{0} \mathrm{CI}$ is consistently calculated to lie at energies below the relevant $S_{1}$ minimum, and could thus provide an alternative radiationless route to the $S_{0}$ state following UV excitation (i.e. could provide another route to photostability).

Radiationless transfer via such a low energy ${ }^{1} \mathrm{CT} / \mathrm{S}_{0} \mathrm{CI}$ was recently suggested as a possible explanation for experimental reports ${ }^{167}$ that the excited state lifetime of adenosine is noticeably shorter than that of bare adenine. The calculated PECs along the O-H. N3 EDPT coordinate of adenosine (Fig. 15(a)) agree well with the previous calculations by Tuna et al. ${ }^{168}$ These show that, following vertical excitation to the 'bright' ${ }^{1} \mathrm{~L}_{\mathrm{b}}$ state, there are barrierless pathways to lower energy CIs with the $\mathrm{S}_{0}$ state in both the $Q_{\text {oop }}$ and EDPT coordinates. The ${ }^{1} \mathrm{CT} / \mathrm{S}_{0} \mathrm{CI}$ is calculated to lie at lower energy and, given that it is reached by intramolecular transfer of a light $\mathrm{H}$ atom, could very well constitute a competitive non-radiative decay pathway following UV excitation. We note the obvious analogy between such a ${ }^{1} \mathrm{CT} / \mathrm{S}_{0} \mathrm{CI}$ in an EDPT coordinate and the previously discussed $1^{1} \pi \sigma^{*} / \mathrm{S}_{0}$ CI in the $R_{\mathrm{O}-\mathrm{H}}$ stretch coordinate in phenols. The former will generally be stabilised (and thus potentially active at lower excitation energies), however, given the presence of an acceptor $\mathrm{O}$ (or $\mathrm{N}$ ) site on the aromatic ring.

The other nucleosides also show a low energy ${ }^{1} \mathrm{CT} / \mathrm{S}_{0} \mathrm{CI}$ in the EDPT coordinate. The calculated PECs leading to the ${ }^{1} \mathrm{CT} / \mathrm{S}_{0}$ CI in guanosine, for example (right hand panel in Fig. 15(b)), have very similar profiles to those for adenosine. So, too, do the respective PECs linking the FC region to the ${ }^{1} \pi \pi^{*} / \mathrm{S}_{0}$ MECI along $Q_{\text {oop }}$ (left hand panel in Fig. 15(b)). The calculated PECs in the $Q_{\text {oop }}$ coordinate show obvious similarities with those of 
the corresponding base (guanine, Fig. 12(b)). Again, the excited state and CI energies are somewhat elevated in the nucleoside reflecting the stabilisation of the ground state by intramolecular H-bonding. As in adenosine, therefore, theory suggests that IC via the ${ }^{1} \mathrm{CT} / \mathrm{S}_{0}$ CI could be a competitive photoprotection pathway following UV photoexcitation of guanosine.

Analogous ${ }^{1} \mathrm{CT} / \mathrm{S}_{0}$ CIs have been identified in the respective EDPT coordinates for 5-methyluridine and cytidine also (Fig. 14(a) and (b)). In the specific case of 5-methyluridine, however, the present calculations return an energy barrier in the PEC linking the FC region and the ${ }^{1} \mathrm{CT} / \mathrm{S}_{0} \mathrm{CI}$, which is also calculated to lie at relatively higher energy than in the other nucleobases. For both 5-methyluridine and cytidine, the PEC along $Q_{\text {oop }}$ leading to the ${ }^{1} \pi \pi^{*} / \mathrm{S}_{0}$ MECI with a prefulvenic geometry is calculated to be barrierless. Based on the present limited set of calculations, it is thus tempting to suggest that: (i) EDPT will play a greater relative role in coupling photoexcited cytidine, adenosine and guanosine molecules back to their respective $S_{0}$ states; and (ii) the non-radiative decay of 5-methyluridine (uridine), and the excited state lifetimes of this nucleoside, should be largely determined by coupling at the ${ }^{1} \pi \pi^{*} / \mathrm{S}_{0}$ CI in $Q_{\text {oop }}$ and thus similar to that of $\mathrm{T}(\mathrm{U})$. Zgierski and Alavi reached broadly similar conclusions in prior computational studies of cytidine and bare cytosine. ${ }^{169}$ However, recent time-resolved pump-probe studies of gas-phase adenosine, cytidine and 5-methyluridine return excited state lifetimes that are, in all cases, shorter than those of the corresponding isolated nucleobase. ${ }^{170}$ This, the authors propose, reflects the additional excited state decay via EDPT enabled by the sugar backbone, which is not present in the isolated nucleobases. ${ }^{169}$ Clearly, there is a case for further, higher level theoretical studies of the EDPT decay path in all of the nucleosides.

\subsection{The DNA and RNA base pairs}

EDPT has also been identified as an efficient deactivation pathway following photoexcitation of WC DNA/RNA base pairs. In the case of the WC A-T base pair, ${ }^{171,172}$ ab initio studies identified a ${ }^{1} \pi \pi^{*}$ CT state arising via electronic promotion from an A-centred $\pi$ orbital to a T-centred $\pi^{*}$ orbital in addition to a number of locally excited (LE) ${ }^{1} n \pi^{*}$ and ${ }^{1} \pi \pi^{*}$ states (i.e. states formed by electron promotion localised on just A or T). Excitations of the former type lead to charge separation, which is countered by excited state proton transfer.

Key PECs along this EDPT coordinate (i.e. extending one of the amine-centred $\mathrm{N}-\mathrm{H}$ bonds attached to $\mathrm{C} 6$ in A towards the $\mathrm{O}$ attached to $\mathrm{C} 4$ in $\mathrm{T}$ ) are shown in Fig. 16(a). Again, the filled black points show the 'relaxed' $S_{0}$ energies calculated by progressively extending this $\mathrm{N}-\mathrm{H}$ driving coordinate and, at each $R_{\mathrm{N}-\mathrm{H}}$ value, allowing all other internal degrees of freedom to relax to the minimum energy configuration. The open coloured points show the various excited state energies calculated at the $\mathrm{S}_{0}$ geometries returned in this way. The filled red points define the 'relaxed' PEC for the ${ }^{1} \mathrm{CT}$ state, and the open black symbols show the corresponding $\mathrm{S}_{0}$ state energies calculated at these relaxed $\mathrm{S}_{1}$ geometries. As Fig. 16(a) shows, the PEC for the ${ }^{1} \mathrm{CT}$ state is barrierless and forms a CI with the $\mathrm{S}_{0}$ PES at extended $R_{\mathrm{N}-\mathrm{H}}$.
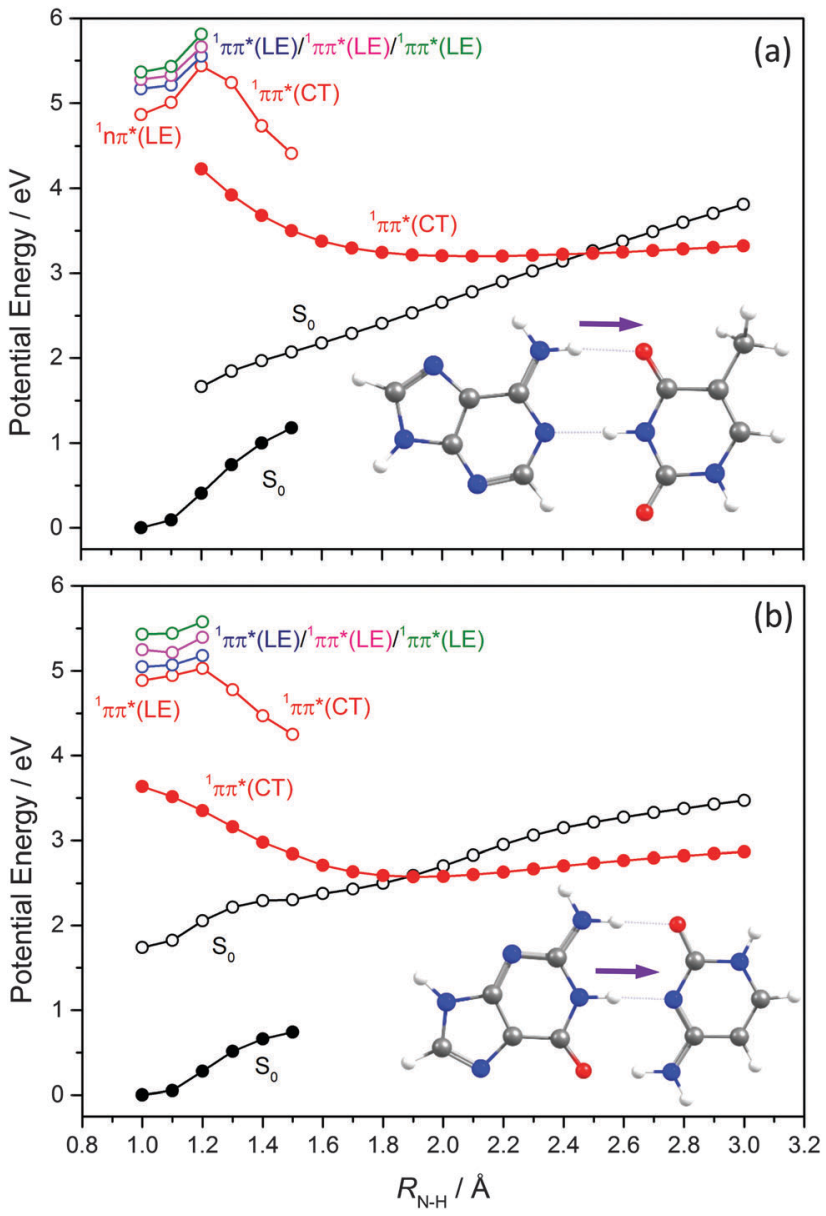

Fig. 16 Potential energy profiles of (a) $A-T$ and (b) $G-C$ base pairs along the EDPT coordinate calculated at ADC2 level of theory. The filled black points show the 'relaxed' $\mathrm{S}_{0}$ energies calculated at each $R_{\mathrm{N}-\mathrm{H}}$ value having allowed all other internal degrees of freedom to relax to the minimum energy configuration. The open coloured points show the excited state energies calculated at these optimised $\mathrm{S}_{0}$ geometries. The filled red points show the 'relaxed' PEC for the ${ }^{1} \mathrm{CT}$ state, while the open black points show the corresponding $\mathrm{S}_{0}$ state energy calculated at each relaxed $\mathrm{S}_{1}$ geometry.

The calculated energy of this MECI is below the VEE of any of the 'bright' LE states. Radiationless transfer to and from this ${ }^{1} \mathrm{CT}$ state thus provides a route by which base pairs initially excited to a ${ }^{1} \pi \pi^{*}$ LE state can return to the $\mathrm{S}_{0}$ state and, following a ground state $\mathrm{H}$ atom transfer, reform the starting base pair structure - thereby constituting another possible route to photostability. Perun et al. ${ }^{170}$ also calculated PECs for the corresponding ${ }^{1} \pi \pi^{*} \mathrm{LE}$ and ${ }^{1} \mathrm{CT}$ states for what is the most stable A-T conformer in the gas phase (characterised by H-bonds between $\mathrm{N} 9-\mathrm{H}$ on A and the $\mathrm{O}=\mathrm{C} 2$ carbonyl group on $\mathrm{T}$, and between $\mathrm{N} 1-\mathrm{H}$ on $\mathrm{T}$ and $\mathrm{N} 3$ on A). The PEC for the ${ }^{1} \mathrm{CT}$ state in this conformer, assuming $R_{\mathrm{N} 9-\mathrm{H}}$ as the driving coordinate, was found at significantly higher energies than the analogous ${ }^{1} \mathrm{CT}$ state in the WC A-T base pair and, crucially, not to form a CI with the $\mathrm{S}_{0}$ PES.

Equivalent studies of the WC G-C base pair identified ${ }^{1} \pi \pi^{*}$ LE states centred on both $\mathrm{G}$ and $\mathrm{C}$ and a low-lying ${ }^{1} \pi \pi^{*} \mathrm{CT}$ state formed by exciting a G-centred $\pi$ electron to a C-centred $\pi^{*}$ orbital. ${ }^{173-177}$ As Fig. 16(b) shows, the PEC for this ${ }^{1} \mathrm{CT}$ state 
calculated using $R_{\mathrm{N} 1-\mathrm{H}}$ as the driving coordinate exhibits a CI with the $\mathrm{S}_{0}$ state. Thus, as with the WC A-T base pair, theory suggests EDPT via a ${ }^{1} \mathrm{CT}$ state could provide an efficient nonradiative route to the $S_{0}$ state after $\mathrm{UV}$ excitation of the $\mathrm{WC} \mathrm{G-C}$ base pair. Again, the calculated PEC for the analogous ${ }^{1} \mathrm{CT}$ state in the most stable (gas phase) conformer of $\mathrm{G}-\mathrm{C}$ (i.e. with $\mathrm{H}$-bonds between $\mathrm{N} 1-\mathrm{H}$ on $\mathrm{G}$ and $\mathrm{O}=\mathrm{C} 2$ on $\mathrm{C}$, and between the $\mathrm{C} 6=\mathrm{O}$ on $\mathrm{G}$ and $\mathrm{N} 1-\mathrm{H}$ on $\mathrm{C}$ ) using the $R_{\mathrm{N} 1-\mathrm{H}}$ bond in $\mathrm{G}$ as the driving coordinate shows no analogous CI with the $\mathrm{S}_{0}$ PES. ${ }^{178}$

Analyses of REMPI spectra of jet-cooled samples of many DNA/RNA base pair structures serve to reinforce these conclusions. The long wavelength UV absorption spectra of those structures assigned as WC conformers are broad and diffuse, whereas the corresponding spectra of all other conformers investigated to date exhibit sharp structure. ${ }^{179,180}$ Solution phase ultrafast transient absorption studies of the $\mathrm{WC} \mathrm{G-C}$ base pair confirm that the spectral breadth is attributable to the short excited state lifetime. ${ }^{181,182}$ All such observations serve to support suggestions that the WC variants of the A-T and $\mathrm{G}-\mathrm{C}$ base pairs have a special status, distinguished by the availability of uniquely efficient ${ }^{1} \mathrm{CT}$ state based deactivation mechanisms that minimise the opportunity for deleterious UV photoinduced chemistry. However, we also note that solution phase transient absorption studies of the WC G-C base pair (in chloroform) suggest a small yield of products arising from a double proton transfer process, wherein one of the $\mathrm{H}$ atoms in the amine group attached to the $\mathrm{C} 4$ atom in the $\mathrm{C}$ moiety in the ${ }^{1} \mathrm{CT}$ state is transferred to the $\mathrm{O}$ atom in the $\mathrm{C} 6=\mathrm{O}$ group in $\mathrm{G} .{ }^{183}$ The net effect of the second (reverse) $\mathrm{H}$ atom transfer is to return population to the $S_{0}$ state, albeit in a different tautomeric form than the starting WC base pair.

The importance of out-of-plane ring deformations in facilitating IC in $\mathrm{N}$ containing heterocycles has been highlighted earlier in this Article. Thus, as for the isolated bases (Fig. 9 and 11), we have used a LIIC approach to calculate PECs along the $Q_{\text {oop }}$ coordinate leading to the MECI between an excited state and the $\mathrm{S}_{0}$ state of the WC A-T base pair. As Fig. 17 shows, the potential energies of the first few singlet excited states generally increase with out-of-plane distortion. However, the PEC for one excited state in the energy range falls with increasing $Q_{\text {oop }}$ and exhibits a CI with the $\mathrm{S}_{0}$ state. Inspecting the various orbital promotions (shown in the ESI, $\dagger$ Fig. S33), we find that the former excited states all arise via $\pi^{*} \leftarrow \mathrm{n}$ or $\pi^{*} \leftarrow \pi$ electron promotions localised on one of the chromophores, whereas the one excited state that displays a very different $Q_{\text {oop }}$ dependence is the first formed by a $\pi^{*} \leftarrow \pi$ excitation involving orbitals that are delocalised (DE) over the entire base pair. The calculated energy of this ${ }^{1} \pi \pi^{*}(\mathrm{DE}) / \mathrm{S}_{0}$ MECI $(\sim 7 \mathrm{eV})$ is much higher than that of the ${ }^{1} \pi \pi^{*} / \mathrm{S}_{0}$ MECIs identified in the corresponding $Q_{\text {oop }}$ coordinate in the isolated A or T bases. This difference can be attributed to the inter-base H-bonding interactions, which serve to restrain the out-of-plane distortion of the individual bases. This digression provides one illustration of the subtle, yet complex, consequences of non-covalent interactions on the relative efficacies of different non-radiative decay pathways: out-ofplane deformations are pivotal in promoting the non-radiative

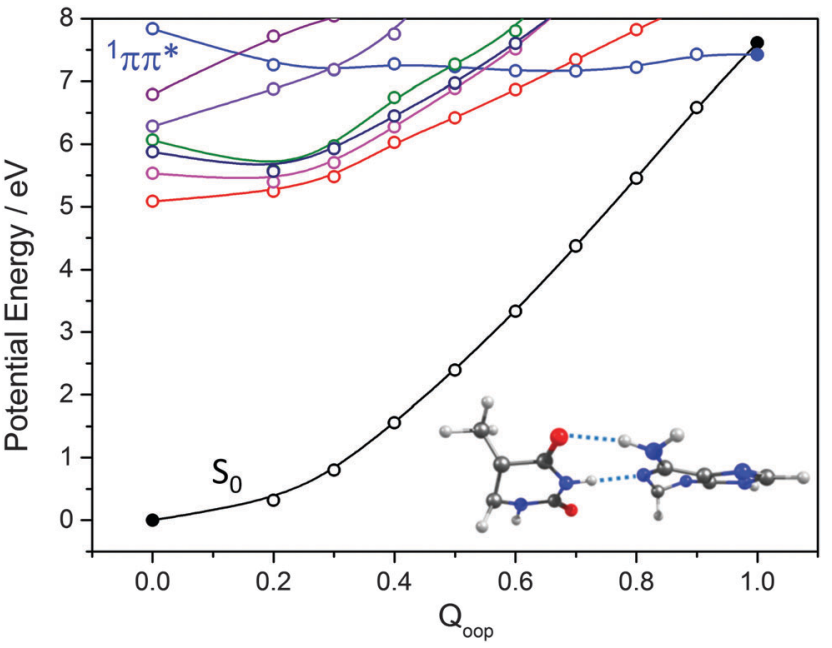

Fig. 17 PECs along $Q_{\text {oop }}$ for the ground and first few singlet excited states of the WC A-T base pair calculated at CASPT2 level of theory. Filled points define the energies of the optimised $S_{0}$ minimum and the prefulvenic ${ }^{1} \pi \pi^{\star} / S_{0}$ $\mathrm{MECl}$, the optimised geometry of which is shown at the lower right.

decay of the excited states of individual DNA bases yet, once noncovalently linked as a WC base pair, the dominant relaxation pathway is generally via CIs located along the EDPT coordinate.

A thorough survey of even a subset of the many other instances where non-covalent interactions have been implicated in the non-radiative decay of stacked base pairs, and single and double stranded DNA, is outside the scope of this article. But we end by highlighting one example that serves to illustrate the potential for competition between base-pairing and base-stacking interactions. Early reports of a longer lived $(\sim 100 \mathrm{ps})$ decay component in transient absorption studies following UV excitation of single and double-stranded oligonucleotides composed of $\mathrm{A}$ and $\mathrm{T}$ units $^{184,185}$ have since been extended and generalised to include other bases and short segments of both single and double stranded DNA. ${ }^{186-193}$ The long lived component is now attributed to excited states arising as a result of photo-induced intrastrand electron transfer (ET), whereby a $\pi$ electron from one base in a stack of nucleosides or base pairs is promoted to a $\pi^{*}$ orbital of an adjacent base in the same stack (i.e. electron hole pair creation in neighbouring stacked DNA bases). The intrastrand ET could then drive interstrand proton transfer from, or to, the base paired with, respectively, the radical anion and the radical cation formed in the ET process - yielding rare tautomers of the original base pair which then decay on a sub-nanosecond timescale. The similarities between this and the classic EDPT mechanism invoked to explain the ultrafast non-radiative decay of electronic excitation in isolated base pairs ${ }^{170,172}$ (illustrated in Fig. 16) are self-evident. So, too, is the difference. The electron and proton implicated in these long-lived excited states originate from neighbouring bases in a stack (rather than the same base in a single base pair).

The PECs shown in Fig. 18 illustrate aspects of this behaviour. We start with a G-C base pair within a stack, and assume that the $\mathrm{C}$ carries a negative charge as a result of photo-induced ET from a neighbouring base (in the strand). This we model as a radicalanion $\mathrm{G}^{-} \mathrm{C}^{-}$pair. Fig. 18(a) shows the relaxed PEC along $R_{\mathrm{N} 1-\mathrm{H}}$ 

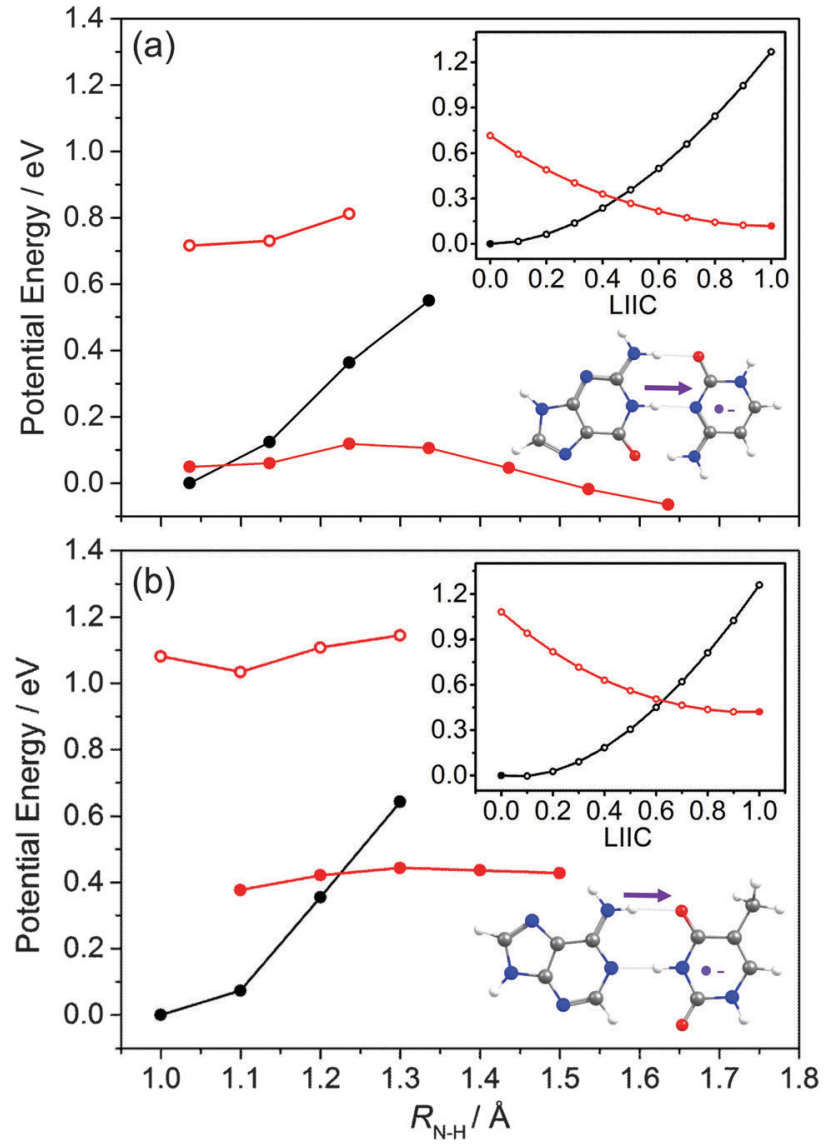

Fig. 18 Calculated PECs along the $\mathrm{N}-\mathrm{H} \cdots \mathrm{N}(\mathrm{N}-\mathrm{H} \cdots \mathrm{O})$ proton transfer coordinate (shown by the arrow on the respective structures at the bottom right) for the neutral and radical-anion forms of the (a) G-C and (b) A-T WC base pair. The relaxed $S_{0}$ state energies are shown as black points, the open red points show the energies of the radical-anion $D_{0}$ state at the corresponding $\mathrm{S}_{0}$ relaxed geometry, and the filled red points show the $\mathrm{D}_{0}$ state energies after relaxing all degrees of freedom apart from the $R_{\mathrm{N}-\mathrm{H}}$ coordinate of interest. The upper inset shows the calculated PECs along a LIIC linking the minimum energy geometries of the $\mathrm{S}_{0}$ state (filled black point) and the $\mathrm{D}_{0}$ state after extending $R_{\mathrm{N}-\mathrm{H}}$ by 0.2 and $0.3 \AA$ (filled red point) for the $\mathrm{G}-\mathrm{C}$ and $\mathrm{A}-\mathrm{T}$ pair, respectively.

(the same driving coordinate as in Fig. 16(b)) for the ground state neutral G-C base pair (solid black points), and PECs for the $\mathrm{G}-\mathrm{C}^{-}$radical-anion pair both at the relevant neutral $\mathrm{S}_{0}$ minimum energy geometries and after allowing all other nuclear degrees of freedom to relax to the minimum energy geometry of its doublet ground $\left(\mathrm{D}_{0}\right)$ state (depicted by open and filled red points, respectively). The inset at the upper right shows the calculated neutral $\left(\mathrm{S}_{0}\right)$ and radical-anion $\left(\mathrm{D}_{0}\right)$ PECs along a LIIC linking the minimum energy geometry of the ground state neutral (at $R_{\mathrm{N} 1-\mathrm{H}}=1.038 \AA$ ) and the minimum energy configuration of the radical-anion at $R_{\mathrm{N} 1-\mathrm{H}}=1.238 \AA$. The calculated PECs show a low energy CI in the $R_{\mathrm{N} 1-\mathrm{H}}$ coordinate associated with proton transfer from the $\mathrm{G}$ to the $\mathrm{C}^{-}$. This process, which the main figure shows to be mildly exothermic, is illustrated on the structure shown at the lower right. Fig. 18(b) shows the corresponding PECs for the neutral A-T base pair and the A-T $\mathrm{T}^{-}$radical-anion along the same $R_{\mathrm{N}-\mathrm{H}}$ coordinate as used in Fig. 16(a). The LIIC used in the upper insert in this case spans the range $1.0 \leq R_{\mathrm{N}-\mathrm{H}} \leq 1.2 \AA$. Though qualitatively similar to those for the G-C base pair (Fig. 18(a)), these PECs show significant differences: the energy of the neutral base pair at its equilibrium geometry is always below that of the radical-anion, and the potential energy of the relaxed $\mathrm{D}_{0}$ PEC if anything increases with increasing $R_{\mathrm{N}-\mathrm{H}}$. These model calculations thus suggest no driving force for proton transfer from A to $\mathrm{T}^{-}$. Such contrasting propensities for proton transfer, from $\mathrm{G}$ to $\mathrm{C}^{-}$but not from $\mathrm{A}$ to $\mathrm{T}^{-}$, accord well with the recent time-resolved vibrational spectroscopy measurements of Zhang et al. ${ }^{193}$

\section{Conclusions}

This Perspective Article reports a systematic analysis of possible non-radiative decay mechanisms following UV photoexcitation of families of nitrogen containing heterocycles. The availability of non-radiative decay mechanisms by which photoexcited molecules can funnel back to their ground state, without experiencing potentially deleterious chemical transformation, is fundamental to molecular photostability. Photostability requires that the rate of internal conversion to the original ground state configuration far exceeds that of any competing population loss process (e.g. fluorescence, isomerisations, reaction, bond fission, etc.). To achieve such 'ultrafast' nonradiative decay from an excited state PES to the ground state, the molecule must be able to sample regions of conical intersection between the PESs. ${ }^{194}$

The present investigation relies on use of electronic structure methods, complemented by a large body of pre-existing experimental and theoretical literature. Starting from phenol and indole, we have explored the structural and photophysical consequences of progressive incorporation of $\mathrm{N}$ atoms into the ring structure. Specific unicyclic systems investigated include selected hydroxypyridines, hydroxypyrimidines and the DNA bases T, U and C. Amongst the bicyclic systems, we have focussed on two azaindoles, purine, A and G. Having identified and rationalised specific photophysical trends in these families of molecular prototypes, we then extended the study to consider some of the additional richness and complexity with regard to excited state non-radiative decay that can arise via non-covalent interactions - in, for example, ribonucleosides, base pairs and stacked bases and base pairs. CIs are shown to be pervasive, but the forms of nuclear distortion required to access the CIs of lowest energy (and thus facilitate non-radiative decay) are seen to be sensitively dependent on the starting molecule, and its immediate environment. Nonetheless, as we now summarise, certain guiding trends and principles clearly emerge from the study.

Addressing first the isolated molecular prototypes, $\mathrm{N}$ incorporation is shown to result in a progressive relative stabilisation of the ground state keto-tautomer ( $c f$. the enol-form), consistent with the observed dominance of keto-tautomers in the DNA/RNA nucleobases, ribonucleosides and WC base pairs. Focussing now 
on excited states, $\mathrm{O}-\mathrm{H}$ bond fission is a common population loss process following UV photoexcitation of phenols. ${ }^{9,17,19,25}$ However, the present work shows that incorporating even one $\mathrm{N}$ atom affords sufficient stabilisation of the ${ }^{1} \pi \pi^{*}$ PES towards a ${ }^{1} \pi \pi^{*} / \mathrm{S}_{0} \mathrm{CI}$ in an out-of-plane deformation coordinate that IC via such prefulvenic distorted structures becomes the dominant (and efficient) decay route following UV excitation of the $\mathrm{N}$-containing unicycles. Such predictions accord with the available excited state lifetime data. The simplest bicyclic system considered in this work, indole, already contains one $\mathrm{N}$ atom. This atom is in the 5-membered ring, however, and the lowest energy ${ }^{1} \pi \pi^{*} / \mathrm{S}_{0} \mathrm{CI}$ in the $Q_{\text {oop }}$ coordinate is calculated to lie well above the $\mathrm{S}_{1}\left({ }^{1} \mathrm{~L}_{\mathrm{b}}\right) \leftarrow \mathrm{S}_{0}$ origin - consistent with prior observations of fluorescence from electronically excited state indole molecules. ${ }^{195}$ The present calculations show that the addition of at least one more $\mathrm{N}$ atom, in the 6-membered ring, is necessary in order to modify the topography of the ${ }^{1} \pi \pi^{*}$ PES sufficiently that distortion along $Q_{\text {oop }}$ to a CI with the $S_{0}$ PES becomes a barrierless, exothermic non-radiative decay pathway. Such trends, identified in both the unicyclic and bicyclic families, are consistent with the well-documented ultrafast excited state decay (and the photostability) of the isolated DNA(RNA) bases T(U), C, A and G.

Understanding the excited state decay dynamics of isolated nucleobases is a necessary but, as the latter part of this Perspective Article shows, not a sufficient prerequisite for unravelling the dominant non-radiative decay mechanism(s) operating when the base is a part of a larger chemical structure. In the nucleosides, for example, one or more heteroatoms in the base may experience $\mathrm{H}$-bonding interactions with $\mathrm{OH}$ groups of the sugar backbone. From the perspective of the respective bases, such interactions are predicted to have only a minor perturbative effect on the ${ }^{1} \pi \pi^{*}$ PES leading to the ${ }^{1} \pi \pi^{*} / \mathrm{S}_{0}$ MECI along $Q_{\text {oop }}$. But the existence of such H-bonds can also promote new, alternative non-radiative decay pathways wherein charge-transfer character induced by the photexcitation causes an $\mathrm{H}$ atom to migrate from the sugar to one of the heteroatoms of the base. The present calculations hint that - particularly in the cases of adenosine, guanosine and cytidine - such an EDPT mechanism could be a competitive route for channeling excited state population back to the $\mathrm{S}_{0}$ state via a CI in the relevant $\mathrm{O}-\mathrm{H} \cdots \mathrm{N}$ coordinate.

$\mathrm{H}$-bonds are central to base pair formation, and have a profound effect on the photophysics of electronically excited base pairs. Isolated base-pairs decay non-radiatively, on an ultrafast timescale. But this decay is not via ${ }^{1} \pi \pi^{*} / \mathrm{S}_{0}$ CIs in the $Q_{\text {oop }}$ coordinate as in the individual bases; the energies of such CIs are substantially raised in the base-pairs by the interbase $\mathrm{H}$-bonds that restrain the out-of-plane distortion of either component base. Rather, the minimum energy decay pathway following UV excitation involves interbase charge-transfer, and subsequent motion through a low energy CI in the EDPT coordinate associated with extending the N6-H bond in A (in the WC A-T base pair) or $\mathrm{N} 1-\mathrm{H}$ in $\mathrm{G}$ (in the $\mathrm{WC} \mathrm{G}-\mathrm{C}$ base pair). The WC structures are not the lowest energy conformers of the isolated base pairs in the gas phase. But equivalent calculations for these minimum energy conformers of A-T and G-C reveal no similar low energy ${ }^{1} \mathrm{CT} / \mathrm{S}_{0}$ CI in the EDPT coordinate, encouraging suggestions that the WC variants are special - by virtue of their having a particularly efficient ${ }^{1} \pi \pi^{*} \mathrm{CT}$ state based deactivation mechanism that minimises the opportunity for potentially UV induced photodamage.

This survey ends with a note of caution. Not only do studies of the isolated bases fail to reveal key aspects of the excited state photophysics displayed by the WC base pairs, but theoretical studies of the isolated base pairs also fail to account for all aspects of the photophysics exhibited by stacks of base pairs. Specifically, several recent experiments identify a longer lifetime component within the excited state decay which theory can explain by invoking a role for photoinduced intrastrand electron transfer, which drives subsequent interstrand proton transfer within a base pair - thereby returning the system to the ground state of (a variant) of the original base pair. ${ }^{183-192}$ Given the evident influence of non-covalent interactions, it is worth reiterating that the calculations reported in this Perspective are all performed under isolated molecule conditions. Many prior absorption studies have shown that solvation affects the absorption spectra (and thus the relative energies of the excited states) of such systems. However, none of the recent time-resolved experiments or theoretical investigations suggest that solvent interactions change the following fundamental conclusion: the excited states of individual DNA/RNA bases, of the WC base pairs, and of assemblies of such base pairs, in the gas phase or in solution, all have access to a sufficiently diverse range of low energy non-radiative decay pathways to ensure rapid and efficient repopulation of the ground state, and thus photostability.

\section{Acknowledgements}

The Bristol based authors are grateful to EPSRC for the award of a Programme Grant (EP/L005913) and to colleagues Prof. Andrew Orr-Ewing and Dr Katharina Röttger for their invaluable feedback on an earlier draft of this manuscript. TNVK is grateful for the award of a TUM University Foundation Fellowship. Additional outputs that underpin data reported in this paper have been placed in the University of Bristol's research data repository and can be accessed using the following DOI: 10.5523/bris.16bza2y6ry2iz14jsuovowv592.

\section{References}

1 A. L. Sobolewski and W. Domcke, Chem. Phys., 2000, 259, 181-191.

2 A. L. Sobolewski, W. Domcke, C. Dedonder-Lardeux and C. Jouvet, Phys. Chem. Chem. Phys., 2002, 4, 1093-1100.

3 M. N. R. Ashfold, B. Cronin, A. L. Devine, R. N. Dixon and M. G. D. Nix, Science, 2006, 321, 1637-1640.

4 K.-L. Han and G.-Z. He, J. Photochem. Photobiol., C, 2007, 8, 55-66. 
5 M. N. R. Ashfold, G. A. King, D. Murdock, M. G. D. Nix, T. A. A. Oliver and A. G. Sage, Phys. Chem. Chem. Phys, 2010, 12, 1218-1238.

6 W. Domcke and D. R. Yarkony, Annu. Rev. Phys. Chem., 2012, 63, 325-352.

7 G. M. Roberts and V. G. Stavros, Chem. Sci., 2014, 5, 1698-1722.

8 Z. Lan, W. Domcke, V. Vallet, A. L. Sobolewski and S. Mahapatra, J. Chem. Phys., 2005, 122, 224315.

9 M. G. D. Nix, A. L. Devine, B. Cronin, R. N. Dixon and M. N. R. Ashfold, J. Chem. Phys., 2006, 125, 133318.

10 A. L. Devine, M. G. D. Nix, B. Cronin and M. N. R. Ashfold, Phys. Chem. Chem. Phys., 2007, 9, 3749-3762.

11 G. A. King, A. L. Devine, M. G. D. Nix, D. E. Kelly and M. N. R. Ashfold, Phys. Chem. Chem. Phys., 2008, 10, 6417-6429.

12 M. N. R. Ashfold, A. L. Devine, R. N. Dixon, G. A. King, M. G D. Nix and T. A. A. Oliver, Proc. Natl. Acad. Sci. U. S. A., 2008, 105, 12701-12706.

13 A. Iqbal, L.-J. Pegg and V. G. Stavros, J. Phys. Chem. A, 2008, 112, 9531-9534.

14 O. P. J. Vieuxmaire, Z. Lan, A. L. Sobolewski and W. Domcke, J. Chem. Phys., 2008, 129, 224307.

15 G. A. King, T. A. A. Oliver, M. G. D. Nix and M. N. R. Ashfold, J. Phys. Chem. A, 2009, 113, 7984-7993.

16 G. A. Pino, A. N. Oldani, E. Marceca, M. Fujii, S.-I. Ishiuchi, M. Miyazaki, M. Broquier, C. Dedonder and C. Jouvet, J. Chem. Phys., 2010, 133, 124313.

17 R. N. Dixon, T. A. A. Oliver and M. N. R. Ashfold, J. Chem. Phys., 2011, 134, 194303.

18 R. A. Livingstone, J. O. F. Thompson, M. Iljina, R. J. Donaldson, B. J. Sussman, M. J. Paterson and D. Townsend, J. Chem. Phys., 2012, 137, 184304.

19 G. M. Roberts, A. S. Chatterley, J. D. Young and V. G. Stavros, J. Phys. Chem. Lett., 2012, 3, 348-352.

20 D. J. Hadden, G. M. Roberts, T. N. V. Karsili, M. N. R. Ashfold and V. G. Stavros, Phys. Chem. Chem. Phys., 2012, 14, 13415-13428.

21 S. G. Ramesh and W. Domcke, Faraday Discuss., 2013, 163, 73-94.

22 X. Xu, K. R. Yang and D. G. Truhlar, J. Chem. Theory Comput., 2013, 9, 3612-3625.

23 T. N. V. Karsili, A. M. Wenge, S. J. Harris, D. Murdock, J. N. Harvey, R. N. Dixon and M. N. R. Ashfold, Chem. Sci., 2013, 4, 2434-2446.

24 S. Albert, P. Lerch, R. Prentner and M. Quack, Angew. Chem., Int. Ed., 2013, 52, 346-349.

25 T. N. V. Karsili, A. M. Wenge, B. Marchetti and M. N. R. Ashfold, Phys. Chem. Chem. Phys., 2014, 16, 588-598.

26 X. Xu, J. Zheng, K. R. Yang and D. G. Truhlar, J. Am. Chem. Soc., 2014, 136, 16378-16386.

27 K. R. Yang, X. Xu, J. Zheng and D. G. Truhlar, Chem. Sci., 2014, 5, 4661-4680.

28 S. J. Harris, T. N. V. Karsili, D. Murdock, T. A. A. Oliver, A. M. Wenge, D. K. Zaouris, M. N. R. Ashfold, J. N. Harvey, J. D. Few, S. Gowrie, G. Hancock, D. J. Hadden,
G. M. Roberts, V. G. Stavros, G. Spighi, L. Poisson and B. Soep, J. Phys. Chem. A, 2015, 119, 6045-6056.

29 A. L. Sobolewski and W. Domcke, Chem. Phys. Lett., 1999, 315, 293-298.

30 B. C. Dian, A. Longarte and T. S. Zwier, J. Chem. Phys., 2003, 6, 2696-2706.

31 H. Lippert, H.-H. Ritze, I. V. Hertel and W. Radloff, Chem. Phys. Lett., 2004, 398, 526-531.

32 M.-F. Lin, C.-M. Tseng, Y. T. Lee and C.-K. Ni, J. Chem. Phys., 2005, 123, 124303.

33 M. G. D. Nix, A. L. Devine, B. Cronin and M. N. R. Ashfold, Phys. Chem. Chem. Phys., 2006, 8, 2610-2618.

34 C. Brand, J. Küpper, D. W. Pratt, W. L. Meerts, D. Krügler, J. Tatchen and M. Schmitt, Phys. Chem. Chem. Phys., 2010, 12, 4968-4979.

35 A. Iqbal and V. G. Stavros, J. Phys. Chem. A, 2010, 114, 68-72.

36 T. A. A. Oliver, G. A. King and M. N. R. Ashfold, Phys. Chem. Chem. Phys., 2011, 13, 14646-14662.

37 R. Livingstone, O. Schalk, A. E. Boguslavskiy, G. Wu, L. T. Bergendahl, A. Stolow, M. J. Paterson and D. Townsend, J. Chem. Phys., 2011, 135, 194307.

38 A. Giussani, M. Merchán, D. Roca-Sanjuán and R. Lindh, J. Chem. Theory Comput., 2011, 7, 4088-4096.

39 R. Montero, A. P. Conde, V. Ovejas, F. Castaño and A. Longarte, J. Phys. Chem. A, 2012, 116, 2698-2703.

40 B. O. Roos, P.-Å. Malmqvist, V. Molina, L. Serrano-Andrés and M. Merchán, J. Chem. Phys., 2002, 116, 7526-7536.

41 F. B. C. Machado and E. R. Davidson, J. Chem. Phys., 1992, 97, 1881-1891.

42 L. Serrano-Andrés, M. P. Fülscher, B. O. Roos and M. Merchán, J. Phys. Chem., 1996, 100, 6484-6491.

43 M. Barbatti, H. Lischka, S. Salzmann and C. M. Marian, J. Chem. Phys., 2009, 130, 034305.

44 D. Voet, W. B. Gratzer, R. A. Cox and P. Doty, Biopolymers, 1963, 1, 193-208.

45 P. R. Callis, Annu. Rev. Phys. Chem., 1983, 34, 329-357.

46 C. E. Crespo-Hernández, B. Cohen, P. M. Hare and B. Kohler, Chem. Rev., 2004, 104, 1977-2019.

47 B. Cohen, C. E. Crespo-Hernández, P. M. Hare and B. Kohler, in Femtochemistry and Femtobiology: Ultrafast Events in Molecular Science, ed. M. Martin and J. T. Hynes, Elsevier, 2004, pp. 463-470.

48 A. L. Sobolewski and W. Domcke, Europhys. News, 2006, 37, 20-23.

49 C. T. Middleton, K. de la Harpe, C. Su, Y. K. Law, C. E. Crespo-Hernández and B. Kohler, Annu. Rev. Phys. Chem., 2009, 60, 217-239.

50 A. L. Sobolewski and W. Domcke, Phys. Chem. Chem. Phys., 2010, 12, 4897-4898.

51 T. Gustavsson, R. Improta and D. Markovitsi, J. Phys. Chem. Lett., 2010, 1, 2025-2030.

52 D. Markovitsi, T. Gustavsson and I. Vayá, J. Phys. Chem. Lett., 2010, 1, 3271-3276.

53 K. Kleinermanns, D. Nachtigallová and M. S. de Vries, Int. Rev. Phys. Chem., 2013, 32, 308-342. 
54 H. Kang, K. T. Lee, B. Jung, Y. J. Ko and S. K. Kim, J. Am. Chem. Soc., 2002, 124, 12958-12959.

55 S. Ullrich, T. Schultz, M. Z. Zgierski and A. Stolow, Phys. Chem. Chem. Phys., 2004, 6, 2796-2801.

56 C. Canuel, M. Mons, F. Piuzzi, B. Tardivel, I. Dimicoli and M. Elhanine, J. Chem. Phys., 2005, 122, 074316.

57 K. Kosma, C. Schröter, E. Samoylova, I. V. Hertel and T. Schultz, J. Am. Chem. Soc., 2009, 131, 16939-16943.

58 J.-W. Ho, H.-C. Yen, W.-K. Chou, C.-N. Weng, L.-H. Cheng, H.-Q. Shi, S.-H. Lai and P.-Y. Cheng, J. Phys. Chem. A, 2011, 115, 8406-8418.

59 M. Kotur, T. C. Weinacht, C. Zhou, K. A. Kistler and S. Matsika, J. Chem. Phys., 2011, 134, 184309.

60 S. Lobsiger, M. A. Trachsel, H.-M. Frey and S. Leutwyler, J. Phys. Chem. A, 2013, 117, 6106-6115.

61 H. Kang, B. Jung and S. K. Kim, J. Chem. Phys., 2003, 118, 6717-6719.

62 S. Ullrich, T. Schultz, M. Z. Zgierski and A. Stolow, J. Am. Chem. Soc., 2004, 126, 2262-2263.

63 H. Satzger, D. Townsend, M. Z. Zgierski, S. Patchkovskii, S. Ullrich and A. Stolow, Proc. Natl. Acad. Sci. U. S. A., 2006, 103, 10196-10201.

64 C. Z. Bisgaard, H. Satzger, S. Ullrich and A. Stolow, ChemPhysChem, 2009, 10, 101-110.

65 H. Kang, J. Chang, S. H. Lee, T. K. Ahn, N. J. Kim and S. K. Kim, J. Chem. Phys., 2010, 133, 154311.

66 D. N. Nikogosyan, D. Angelov, B. Soep and L. Lindqvist, Chem. Phys. Lett., 1996, 252, 322-326.

67 A. Reuther, H. Iglev, R. Laenen and A. Laubereau, Chem. Phys. Lett., 2000, 325, 360-368.

68 J.-M. L. Pecourt, J. Peon and B. Kohler, J. Am. Chem. Soc., 2000, 122, 9348-9349.

69 T. Gustavsson, A. Sharonov and D. Markovitsi, Chem. Phys. Lett., 2002, 351, 195-200.

70 T. Gustavsson, A. Sharonov, D. Onidas and D. Markovitsi, Chem. Phys. Lett., 2002, 356, 49-54.

71 A. Sharonov, T. Gustavsson, V. Carré, E. Renault and D. Markovitsi, Chem. Phys. Lett., 2003, 380, 173-180.

72 B. Cohen, P. Hare and B. Kohler, J. Am. Chem. Soc., 2003, 125, 13594-13601.

73 R. J. Malone, A. M. Miller and B. Kohler, Photochem. Photobiol., 2003, 77, 158-164.

74 T. Gustavsson, Á. Bányász, E. Lazzarotto, D. Markovitsi, G. Scalmani, M. J. Frisch, V. Barone and R. Improta, J. Am. Chem. Soc., 2006, 128, 607-619.

75 T. Gustavsson, N. Sarkar, E. Lazzarotto, D. Markovitsi and R. Improta, Chem. Phys. Lett., 2006, 429, 551-557.

76 G. M. Roberts, H. J. B. Marroux, M. P. Grubb, M. N. R. Ashfold and A. J. Orr-Ewing, J. Phys. Chem. A, 2014, 118, 11211-11225.

77 F. Buchner, A. Nakayama, S. Yamazaki, H.-H. Ritze and A. Lübcke, J. Am. Chem. Soc., 2015, 137, 2931-2938.

78 S. Matsika, J. Phys. Chem. A, 2004, 108, 7584-7590.

79 M. Z. Zgierski, S. Patchkovskii, T. Fujiwara and E. C. Lim, J. Phys. Chem. A, 2005, 109, 9384-9387.

80 S. Perun, A. L. Sobolewski and W. Domcke, J. Phys. Chem. A, 2006, 110, 13238-13244.
81 N. Ismail, L. Blancafort, M. Olivucci, B. Kohler and M. A. Robb, J. Am. Chem. Soc., 2002, 124, 6818-6819.

82 L. Blancafort, B. Cohen, P. M. Hare, B. Kohler and M. A. Robb, J. Phys. Chem. A, 2005, 109, 4431-4436.

83 K. A. Kistler and S. Matsika, J. Phys. Chem. A, 2007, 111, 2650-2661.

84 L. Blancafort, Photochem. Photobiol., 2007, 83, 603-610.

85 M. Merchán, R. González-Luque, T. Climent, L. SerranoAndrés, E. Rodríguez, M. Reguero and D. Peláez, J. Phys. Chem. B, 2006, 110, 26471-26476.

86 G. Zechmann and M. Barbatti, J. Phys. Chem. A, 2008, 112, 8273-8279.

87 D. Asturiol, B. Lasorne, M. A. Robb and L. Blancafort, J. Phys. Chem. A, 2009, 113, 10211-10218.

88 S. Yamazaki, W. Domcke and A. L. Sobolewski, J. Phys. Chem. A, 2008, 112, 11965-11968.

89 Z. Lan, E. Fabiano and W. Thiel, ChemPhysChem, 2009, 10, 1225-1229.

90 K. A. Kistler and S. Matsika, Phys. Chem. Chem. Phys., 2010, 12, 5024-5031.

91 M. Barbatti, A. J. A. Aquino, J. J. Szymczak, D. Nachtigallova, P. Hobza and H. Lischka, Proc. Natl. Acad. Sci. U. S. A., 2010, 107, 21453-21458.

92 V. B. Delchev, A. L. Sobolewski and W. Domcke, Phys. Chem. Chem. Phys., 2010, 12, 5007-5015.

93 M. Barbatti, J. J. Szymczak, A. J. A. Aquino, D. Nachtigallova and H. Lischka, J. Chem. Phys., 2011, 134, 014304.

94 D. Nachtigallová, A. J. A. Aquino, J. J. Szymczak, M. Barbatti, P. Hobza and H. Lischka, J. Phys. Chem. A, 2011, 115, 5247-5255.

95 C. M. Marian, J. Chem. Phys., 2005, 122, 104314.

96 S. Perun, A. L. Sobolewski and W. Domcke, J. Am. Chem. Soc., 2005, 127, 6257-6265.

97 L. Serrano-Andrés, M. Merchán and A. C. Borin, Chem. Eur. J., 2006, 12, 6559-6571.

98 M. Barbatti and H. Lischka, J. Am. Chem. Soc., 2008, 130, 6831-6839.

99 I. Conti, M. Garavelli and G. Orlandi, J. Am. Chem. Soc., 2009, 131, 16108-16118.

100 M. Barbatti, Z. Lan, R. Crespo-Otero, J. J. Szymczak, H. Lischka and W. Thiel, J. Chem. Phys., 2012, 137, 22 A503.

101 J. Petersen, M. Wohlgemuth, B. Sellner, V. BonačićKoutecký, H. Lischka and R. Mitrić, Phys. Chem. Chem. Phys., 2012, 14, 4687-4694.

102 B. P. Fingerhut, K. E. Dorfman and S. Mukamel, J. Phys. Chem. Lett., 2013, 4, 1933-1942.

103 C. G. Triandafillou and S. Matsika, J. Phys. Chem. A, 2013, 117, 12165-12174.

104 F. Plasser, R. Crespo-Otero, M. Pederzoli, J. Pittner, H. Lischka and M. Barbatti, J. Chem. Theory Comput., 2014, 10, 1395-1405.

105 C. M. Marian, J. Phys. Chem. A, 2007, 111, 1545-1553.

106 S. Yamazaki and W. Domcke, J. Phys. Chem. A, 2008, 112, 7090-7097.

107 L. Serrano-Andrés, M. Merchán and A. C. Borin, J. Am. Chem. Soc., 2008, 130, 2473-2484. 
108 M. J. Frisch, G. W. Trucks, H. B. Schlegel, G. E. Scuseria, M. A. Robb, J. R. Cheeseman, G. Scalmani, V. Barone, B. Mennuchi and G. Petersson, et al., Gaussian 09, revision B.01, Gaussian Inc., Wallingford, CT, 2010.

109 T. H. Dunning, Jr., J. Chem. Phys., 1989, 90, 1007-1023.

110 W. J. Hehre, R. F. Stewart and J. A. Pople, J. Chem. Phys., 1969, 51, 2657-2664.

111 H.-J. Werner, P. J. Knowles, G. Knizia, F. R. Manby and M. Schütz, et al., MOLPRO, version 2010.1, a package of ab initio programs, University of Cardiff, Cardiff, UK, 2010, see http:/www.molpro.net.

112 Karlsruhe GmbH, 1989-2007, TURBOMOLE GmbH, since 2007, available from www.turbomole.com.

113 L. D. Hatherley, R. D. Brown, P. D. Godfrey, A. P. Pierlot, W. Caminati, D. Damiani, S. Melandri and L. B. Favero, J. Phys. Chem., 1993, 97, 46-51.

114 R. H. Cox and A. A. Bother-By, J. Phys. Chem., 1969, 73, 2465-2468.

115 M. Kuzuya, A. Noguchi and T. Okuda, J. Chem. Soc., Chem. Commun., 1984, 100, 435-436.

116 R. Sanchez, B. M. Giuliano, S. Melandri, L. B. Favero and W. Caminati, J. Am. Chem. Soc., 2007, 129, 6287-6290.

117 B. M. Giuliano, V. Feyer, K. C. Prince, M. Coreno, L. Evangelisti, S. Melandri and W. Caminati, J. Phys. Chem. A, 2010, 114, 12725-12730.

118 R. D. Brown, P. D. Godfrey, D. McNaughton and A. P. Pierlot, J. Am. Chem. Soc., 1988, 110, 2329-2330.

119 R. D. Brown, P. D. Godfrey, D. McNaughton and A. P. Pierlot, J. Am. Chem. Soc., 1989, 111, 2308-2310.

120 E. Nir, M. Muller, L. I. Grace and M. S. de Vries, Chem. Phys. Lett., 2002, 355, 59-64.

121 E. Nir, I. Hünig, K. Kleinermanns and M. S. de Vries, Phys. Chem. Chem. Phys., 2003, 5, 4780-4785.

122 V. Feyer, O. Plekan, R. Richter, M. Coreno, G. Vall-Ilosera, K. C. Prince, A. B. Trofimov, I. L. Zatseva, T. E. Moskovskaya, E. V. Gromov and J. Schirmer, J. Phys. Chem. A, 2009, 113, 5736-5742.

123 M. Szczesniak, K. Szczepaniak, J. S. Kwiatkowski, K. KuBulat and W. B. Person, J. Am. Chem. Soc., 1988, 110, 8319-8330.

124 G. Bazsó, G. Tarczay, G. Fogarasi and P. G. Szalay, Phys. Chem. Chem. Phys., 2011, 13, 6799-6807.

125 M. Dreyfus, O. Bensaude, G. Dodin and J. E. Dubois, J. Am. Chem. Soc., 1976, 98, 6338-6349.

126 C. Colominas, F. J. Luque and M. Orozco, J. Am. Chem. Soc., 1996, 118, 6811-6821.

127 R. Kobayashi, J. Phys. Chem., 1998, 102, 10813-10817.

128 S. A. Trygubenko, T. V. Bogdan, M. Rueda, M. Orozco, F. J. Luque, J. Sponer, P. Slavicek and P. Hobza, Phys. Chem. Chem. Phys., 2002, 4, 4192-4203.

129 M. Hanus, M. Kabeláč, J. Rejnek, F. Ryjáček and P. Hobza, J. Phys. Chem. B, 2004, 108, 2087-2097.

130 J. Lin, C. Yu, S. Peng, I. Akiyama, K. Li, L. K. Lee and P. R. LeBreton, J. Am. Chem. Soc., 1980, 102, 4627-4631.

131 R. D. Brown, P. D. Godfrey, D. McNaughton and A. P. Pierlot, Chem. Phys. Lett., 1989, 156, 61-63.
132 C. Plützer, E. Nir, M. S. de Vries and K. Kleinermanns, Phys. Chem. Chem. Phys., 2001, 3, 5466-5469.

133 C. Plützer and K. Kleinermanns, Phys. Chem. Chem. Phys., 2002, 4, 4877-4882.

134 M. J. Nowak, L. Lapinski, J. S. Kwiatkowski and J. Leszczyński, J. Phys. Chem., 1996, 100, 3527-3534.

135 M. Dreyfus, G. Dodin, O. Bensaude and J. E. Dubois, J. Am. Chem. Soc., 1975, 97, 2369-2376.

136 M.-T. Chenon, R. J. Pugmire, D. M. Grant, R. P. Panzica and L. B. Townsend, J. Am. Chem. Soc., 1975, 97, 4636-4642.

137 N. C. Gonnella, H. Nakanishi, J. B. Holtwick, D. S. Horowitz, K. Kanamori, N. J. Leonard and J. D. Roberts, J. Am. Chem. Soc., 1983, 105, 2050-2055.

138 A. Laxer, D. T. Major, H. E. Gottlieb and B. Fischer, J. Org. Chem., 2001, 66, 5463-5481.

139 S. K. Mishra, M. K. Shukla and P. C. Mishra, Spectrochim. Acta, Part A, 2000, 56, 1355-1384.

140 L. M. Salter and G. M. Chaban, J. Phys. Chem. A, 2002, 106, 4251-4256.

141 T.-K. Ha, H.-J. Keller, R. Gunde and H.-H. Gunthard, J. Phys. Chem. A, 1999, 103, 6612-6623.

142 K. Szczepaniak and M. Szczesniak, J. Mol. Struct., 1987, 156, 29-42.

143 F. Piuzzi, M. Mons, I. Dimicoli, B. Tardivel and Q. Zhao, Chem. Phys., 2001, 270, 205-214.

144 M. Mons, I. Dimicoli, F. Piuzzi, B. Tardivel and M. Elhanine, J. Phys. Chem. A, 2002, 106, 5088-5094.

145 M. Mons, F. Piuzzi, I. Dimicoli, L. Gorb and J. Leszczynski, J. Phys. Chem. A, 2006, 110, 10921-10924.

146 K. Seefeld, R. Brause, T. Häber and K. Kleinermanns, J. Phys. Chem. A, 2007, 111, 6217-6221.

147 M. Hanus, F. Ryjáček, M. Kabelaáč, T. Kubař, T. V. Bogdan, S. A. Trygubenko and P. Hobza, J. Am. Chem. Soc., 2003, 125, 7678-7688.

148 L. Gorb, A. Kaczmarek, A. Gorb, A. J. Sadlej and J. Leszczynski, J. Phys. Chem. B, 2005, 109, 13770-13776.

149 S. Kato, J. Chem. Phys., 1988, 88, 3045-3056.

150 I. J. Palmer, I. N. Ragazos, F. Bernardi, M. Olivucci and M. A. Robb, J. Am. Chem. Soc., 1993, 115, 673-682.

151 A. L. Sobolewski, C. Woywod and W. Domcke, J. Chem. Phys., 1993, 98, 5627-5641.

152 F. Zhang, Y.-J. Ai, Y. Luo and W.-H. Fang, J. Chem. Phys., 2009, 130, 144315.

153 A. L. Sobolewski, Chem. Phys. Lett., 1993, 211, 293-299.

154 A. L. Sobolewski and L. Adamowicz, J. Phys. Chem., 1996, 100, 3933-3941.

155 H. Ozeki, M. C. R. Cockett, K. Okayama, M. Takahashi and K. Kimura, J. Phys. Chem., 1995, 99, 8608-8612.

156 D. Lee, S. J. Baek, K.-W. Choi, Y. S. Choi and S. K. Kim, Bull. Korean Chem. Soc., 2002, 23, 277-280.

157 A. Held, B. B. Champagne and D. W. Pratt, J. Chem. Phys., 1991, 95, 8732-8743.

158 M. R. Nimlos, D. F. Kelley and E. R. Bernstein, J. Phys. Chem., 1989, 93, 643-651.

159 S. Maeda, Y. Harabuchi, T. Taketsugu and K. Morokuma, J. Phys. Chem. A, 2014, 118, 12050-12058. 
160 D. Nachtigallová, H. Lischka, J. J. Szymczak, M. Barbatti, P. Hobza, Z. Gengeliczki, G. Pino, M. P. Callahane and M. S. de Vries, Phys. Chem. Chem. Phys., 2010, 12, 4924-4933.

161 M. G. D. Nix, A. L. Devine, B. Cronin and M. N. R. Ashfold, J. Chem. Phys., 2007, 126, 124312.

162 A. Z. Britten and G. Lockwood, Spectrochim. Acta, Part A, 1976, 32, 1335-1338.

163 V. Ludwig, Z. M. da Costa, M. S. do Amaral, A. C. Borin, S. Canuto and L. Serrano-Andrés, Chem. Phys. Lett., 2010, 492, 164-169.

164 J. Hager and S. C. Wallace, J. Phys. Chem., 1983, 87, 2121-2127.

165 Y. Huang and M. Sulkes, Chem. Phys. Lett., 1996, 254, 242-248.

166 M. S. de Vries, Top. Curr. Chem., 2014, 355, 33-56.

167 H. Asami, K. Yagi, M. Ohba, S.-H. Urashima and H. Saigusa, Chem. Phys., 2013, 419, 84-89.

168 D. Tuna, A. L. Sobolewski and W. Domcke, J. Phys. Chem. A, 2014, 118, 122-127.

169 M. Z. Zgierski and S. Alavi, Chem. Phys. Lett., 2006, 426, 398-404.

170 S. De Camillis, J. Miles, G. Alexander, O. Ghafur, I. D. Williams, D. Townsend and J. B. Greenwood, Phys. Chem. Chem. Phys., 2015, 17, 23643-23650.

171 S. Perun, A. L. Sobolewski and W. Domcke, J. Phys. Chem. A, 2006, 110, 9031-9038.

172 J. P. Gobbo, V. Saurí, D. Roca-Sanjuán, L. Serrano-Andrés, M. Merchán and A. C. Borin, J. Phys. Chem. B, 2012, 116, 4089-4097.

173 A. L. Sobolewski and W. Domcke, Phys. Chem. Chem. Phys., 2004, 6, 2763-2771.

174 P. R. Markwick and N. L. Doltsinis, J. Chem. Phys., 2007, 126, 175102.

175 G. Groenhof, L. V. Schäfer, M. Boggio-Pasqua, M. Goette, H. Grubmüller and M. A. Robb, J. Am. Chem. Soc., 2007, 129, 6812-6819.

176 V. Saurí, J. P. Gobbo, J. J. Serrano-Pérez, M. Lundberg, P. B. Coto, L. Serrano-Andrés, A. C. Borin, R. Lindh, M. Merchán and D. Roca-Sanjuán, J. Chem. Theory Comput., 2013, 9, 481-496.

177 S. Yamazaki and T. Taketsugu, Phys. Chem. Chem. Phys., 2012, 14, 8866-8877.

178 A. L. Sobolewski, W. Domcke and C. Hättig, Proc. Natl. Acad. Sci. U. S. A., 2005, 102, 17903-17906.

179 E. Nir, C. Plützer, K. Kleinermanns and M. S. de Vries, Eur. Phys. J. D, 2002, 20, 317-329.

180 A. Abo-Riziq, L. Grace, E. Nir, M. Kabelac, P. Hobza and M. S. de Vries, Proc. Natl. Acad. Sci. U. S. A., 2005, 102, 20-23.

181 N. K. Schwalb and F. Temps, J. Am. Chem. Soc., 2007, 129, 9272-9273.

182 K. Röttger and F. Temps, in Ultrafast Phenomena XIX, ed. I. Yamanouchi, S. Cundiff and R. de Vivie Riedle, 2015, vol. 162, pp. 514-516.
183 K. Röttger, H. J. B. Marroux, M. P. Grubb, P. M. Coulter, H. Böhnke, A. S. Henderson, M. C. Galan, F. Temps, A. J. Orr-Ewing and G. M. Roberts, Angew. Chem., Int. Ed., 2015, 54, 14719-14722.

184 C. Crespo-Hernández, B. Cohen and B. Kohler, Nature, 2005, 436, 1141-1144.

185 W.-M. Kwok, C. Ma and D. L. Phillips, J. Am. Chem. Soc., 2006, 128, 11894-11905.

186 T. Takaya, C. Su, K. de la Harpe, C. E. Crespo-Hernández and B. Kohler, Proc. Natl. Acad. Sci. U. S. A., 2008, 105, 10285-10290.

187 K. de la Harpe and B. Kohler, J. Phys. Chem. Lett., 2011, 2, 133-138.

188 I. Vaya, T. Gustavsson, T. Douki, Y. Berlin and D. Markovitsi, J. Am. Chem. Soc., 2012, 134, 11366-11368.

189 G. W. Doorley, M. Wojdyla, G. W. Watson, M. Towrie, A. W. Parker, J. M. Kelly and S. J. Quinn, J. Phys. Chem. Lett., 2013, 4, 2739-2744.

190 C. Su, C. T. Middleton and B. Kohler, J. Phys. Chem. B, 2012, 116, 10266-10274.

191 J. Chen, A. K. Thazhathveetil, F. D. Lewis and B. Kohler, J. Am. Chem. Soc., 2013, 136, 10290-10293.

192 D. B. Bucher, A. Schlueter, T. Carell and W. Zinth, Angew. Chem., Int. Ed., 2014, 55, 11366-11369.

193 Y. Zhang, K. de la Harpe, A. A. Beckstead, R. Improta and B. Kohler, J. Am. Chem. Soc., 2015, 137, 7059-7062.

194 W. Domcke, D. R. Yarkony and H. Koppel, Conical Intersections: Theory, Computation and Experiment, vol. 17 of Advances Series in Physical Chemistry, 2011, and references therein.

195 D. M. Sammeth, S. Yan, L. H. Spangler and P. R. Callis, J. Phys. Chem., 1990, 94, 7340-7342.

196 Y. Matsuda, T. Ebata and N. Mikami, J. Chem. Phys., 1999, 110, 8397-8407.

197 Y. Matsuda, T. Ebata and N. Mikami, J. Chem. Phys., 2000, 113, 573-580.

198 L. B. Clark, G. G. Peschel and I. Tinoco, Jr., J. Phys. Chem., 1965, 69, 3615-3618.

199 H. De Voe and I. Tinoco, Jr., J. Mol. Biol., 1962, 4, 500-517. 200 B. J. Fender, D. M. Sammeth and P. R. Callis, Chem. Phys. Lett., 1995, 239, 31-37.

201 K. Fuke, H. Yoshiuchi and K. Kaya, J. Phys. Chem., 1984, 88, 5840-5844.

202 S. K. Kim and E. R. Bernstein, J. Phys. Chem., 1990, 94, 3531-3539.

203 C. Kang, J. T. Yi and D. W. Pratt, J. Chem. Phys., 2005, 123, 094306.

204 K. Sakota and H. Sekiya, J. Phys. Chem. A, 2009, 113, 2663-2665.

205 M. Schneider, T. Hain and I. Fischer, ChemPhysChem, 2009, 10, 634-636.

206 N. J. Kim, G. Jeong, Y. S. Kim, J. Sung, S. K. Kim and Y. D. Park, J. Chem. Phys., 2000, 113, 10051-10055.

207 E. Nir, C. Janzen, P. Imhof, K. Kleinermanns and M. S. de Vries, J. Chem. Phys., 2001, 115, 4604-4611. 